\title{
Radiatively driven convection in an ice-covered lake investigated by using temperature microstructure technique
}

\author{
Tobias Jonas, ${ }^{1,2}$ Arkady Y. Terzhevik, ${ }^{3}$ Dmitrii V. Mironov, ${ }^{4,5}$ and Alfred Wüest ${ }^{1}$ \\ Received 22 January 2002; revised 31 July 2002; accepted 22 August 2002; published 12 June 2003.
}

[1] Convection in an ice-covered lake, driven by the absorption of solar radiation, is investigated by means of temperature microstructure technique. This type of convection typically occurs in spring, when melting snow on the ice cover enables solar radiation to penetrate into the water body. The diurnal dynamics of the stratification system of five distinct layers is analyzed by means of consecutive CTD profiles and with the aid of a onedimensional model. The model solves the transfer equation of heat and salinity and includes convective procedures to react on density instabilities. This study is focused on the turbulent kinetic energy (TKE) balance. The stratification analysis reveals the importance of several processes for the TKE balance, namely: (1) the entrainment into the top layer from the convective layer below, (2) the inflow of water from melted ice, and (3) the volumetric solar heating. Enabled by the analysis of the temperature microstructure profiles, two TKE budgets are presented. The temporally averaged budget reveals a vertical distribution of generation and dissipation rate similar to the case of coolinginduced convection in a surface boundary layer. But contrary to this reference regime, a transition layer was found in the upper convective layer, where both rates drop back to zero toward the layer above. The second TKE budget is spatially averaged over the convective layer but resolves the diurnal dynamics. The generation rate and dissipation rate feature similar diurnal dynamics, where the dissipation lags on average by 1.5 hours. The temporal change rate of TKE was found to be on the same order of magnitude as the generation rate and the dissipation rate, while the export rate of TKE out of the convective layer was found to be less significant. INDEX TERMS: 4568 Oceanography: Physical: Turbulence, diffusion, and mixing processes; 4524 Oceanography: Physical: Fine structure and microstructure; 4239 Oceanography: General: Limnology; 1845 Hydrology: Limnology; KEYWORDS: convection, turbulence, temperature microstructure, ice-covered lake, limnology, turbulent kinetic energy

Citation: Jonas, T., A. Y. Terzhevik, D. V. Mironov, and A. Wüest, Radiatively driven convection in an ice-covered lake investigated by using temperature microstructure technique, J. Geophys. Res., 108(C6), 3183, doi:10.1029/2002JC001316, 2003.

\section{Introduction}

[2] In spring, when a lake's ice and snow cover melt away, solar radiation begins to penetrate into the water body. The absorption of this radiation may cause convection where the water column is cooled below the temperature of maximum density. One of the first people to suggest convection under such conditions to be driven by the absorption of solar radiation was Birge [1910]. Several studies of photographs of cell-like patterns in the melting ice cover of lakes followed around 1950 [e.g., Brunt, 1946;

\footnotetext{
${ }^{1}$ Swiss Federal Institute of Environmental Science and Technology, Dübendorf and Kastanienbaum, Switzerland.

${ }^{2}$ Now at Alpine Environment Section, Swiss Federal Institute for Snow and Avalanche Research, Davos Dorf, Switzerland.

${ }^{3}$ Northern Water Problems Research Institute, Russian Academy of Sciences, Petrozavodsk, Russia.

${ }^{4}$ German Weather Service, Offenbach am Main, Germany.

${ }^{5}$ Also at Alfred Wegener Institute for Polar and Marine Research, Bremerhaven, Germany.

Copyright 2003 by the American Geophysical Union. 0148-0227/03/2002JC001316
}

Neumann, 1958]. Woodcock [1965] later added temperature records of an ice-covered lake to the discussion, and concluded that the absorption of radiation was causing the convection rather than bottom heating. Barnes and Hobbie [1960] considered the structure of a stably stratified top layer (TL), which separates the convective layer (CL) from the underside of the ice. Results from solving the heat transfer equation balancing radiative heating and molecular diffusion were consistent with their measurements. The first systematic study of radiatively driven convection in an icecovered lake was presented by Farmer [1975]. Records of thermistor strings revealed the evolution of the temperature stratification, indicating convective mixing and penetrative entrainment. His mixed layer model was followed by several other modeling studies [e.g., Petrov and Sutyrin, 1984; Bengtsson, 1996; Mironov et al., 2002].

[3] Since 1995, three shallow Karelian lakes have been under regular investigation, two of which experience a short, strong convection period almost every spring [Malm et al., 1997a, 1997b]. To the best of the authors' knowledge, for the first time direct measurements of microscale temperature fluctuations have been performed during a convection 
period in an ice-covered lake. These measurements took place in spring 1999 at Lake Vendyurskoe (NW-Russia), the largest of the three Karelian lakes mentioned. The temperature microstructure data have been evaluated by means of the Batchelor method [Batchelor, 1959] to estimate the dissipation rate $\epsilon$ of turbulent kinetic energy (TKE). Moreover, the stratification dynamics has been analyzed with particular regard to mechanisms that govern the generation rate of available potential energy (APE), which is transferable into TKE. This allowed assembling a TKE budget, the main focus of this study.

[4] In section 2, a one-dimensional model is presented which solves the transfer equation of heat and salinity balancing radiative heating, molecular diffusion, and turbulent transport. The latter term is dealt with by rearranging the water column during each time step; this according to several different convective procedures, the simplest of which adiabatically imposes a stable density stratification. To obtain information about the buoyancy flux driving the convection, we evaluate the absorption of solar radiation underneath the ice (section 3). Besides the surface radiation flux (radiative heat flux through the underside of the ice), an appropriate parameterization of the vertical absorption pattern is required. Particularly for the uppermost layer, the model helps to optimize the parameterization. Another important effect turns out to be the inflow of water from melted ice, which we assess on the basis of a heat budget of the ice cover and indirect measurements of the cover's thickness. The diurnal dynamics of the stratification is analyzed by means of the temperature microstructure data, CTD data, and model calculations (section 4). Emphasis is placed on the development of the CL boundaries. Their dynamics trace interactions between convective flow and the bordering stratification. Where separable, the specific influence of the salinity and of the temperature stratification, respectively, is considered. The discussion (section 5) begins with a systematic determination of the fraction of the surface radiation flux that is available to generate TKE. The transfer equation of heat is utilized to directly determine the generation rate of TKE from the consecutive temperature microstructure profiles. On the basis of these evaluations and estimates of the dissipation rate of TKE, two different TKE budgets are described in section 5.2. First, a temporally averaged budget reveals vertical profiles of the budget terms. The findings are compared to large-eddy simulation (LES) results from shear-free convective boundary layers. Second, a TKE budget is presented, which is averaged over the CL but resolves the diurnal dynamics of the budget terms. As the steady state assumption turns out to be inappropriate for the scaling of individual profiles of the dissipation rate, we suggest an alternative scaling concept involving the observed time lag between generation and dissipation of TKE.

[5] To simplify the discussion, the notation used in the following is specified in Table 1. For further reference, the water column is subdivided into distinct layers in section 4 .

\section{Data Acquisition and Methods}

\subsection{Site and Experimental Setup}

[6] Lake Vendyurskoe is a small mesotrophic lake of glacial origin. With a length of approximately $7 \mathrm{~km}$, a width
Table 1. Notation

\begin{tabular}{|c|c|c|}
\hline Property & Unit & Description \\
\hline & & stratification layers \\
\hline TL & & top layer, $\mathrm{TL}=\mathrm{CDL}+\mathrm{DL}$ \\
\hline CDL & & conduction layer \\
\hline DL & & diffusive layer \\
\hline $\mathrm{CL}$ & & convective layer \\
\hline EL & & entrainment layer \\
\hline QL & & quiescent layer \\
\hline$z_{0}$ & $\mathrm{~m}$ & depth of interface CDL/DL \\
\hline$z_{1}$ & $\mathrm{~m}$ & depth of interface DL/CL \\
\hline$z_{2}$ & $\mathrm{~m}$ & depth of interface $\mathrm{CL} / \mathrm{EL}$ \\
\hline$z_{3}$ & $\mathrm{~m}$ & depth of interface EL/QL \\
\hline & & TKE budget \\
\hline $\begin{array}{l}\text { APE } \\
F\end{array}$ & $\mathrm{~m}_{3}^{2} \mathrm{~s}_{-3}^{-2}$ & $\begin{array}{l}\text { available potential energy } \\
\text { vertical transport rate of TKE }\end{array}$ \\
\hline & $\begin{array}{l}\mathrm{m}^{3} \mathrm{~s}^{-3} \\
\mathrm{~m}^{2} \mathrm{~s}^{-3}\end{array}$ & $\begin{array}{l}\text { vertical transport rate of TKE } \\
\text { buoyancy flux }\end{array}$ \\
\hline$J_{0}$ & $\mathrm{~m}^{2} \mathrm{~s}^{-3}$ & surface buoyancy flux, $J_{0}=J(z=0)$ \\
\hline$J^{\mathrm{APE}}$ & $\mathrm{m}^{2} \mathrm{~s}^{-3}$ & generation rate of APE \\
\hline$J^{\mathrm{TKE}}$ & $\mathrm{m}^{2} \mathrm{~s}^{-3}$ & generation rate of TKE \\
\hline$Q$ & $\mathrm{~K} \mathrm{~m} \mathrm{~s}^{-1}$ & vertical turbulent temperature flux \\
\hline TKE & $\mathrm{m}^{2} \mathrm{~s}^{-2}$ & turbulent kinetic energy \\
\hline$x$ & $\mathrm{~K}^{2} \mathrm{~s}^{-1}$ & dissipation rate of $T$ variance \\
\hline$\varepsilon$ & $\mathrm{m}^{2} \mathrm{~s}^{-3}$ & dissipation rate of TKE \\
\hline$\gamma$ & $\mathrm{m}^{2} \mathrm{~s}^{-3}$ & temporal change rate of TKE \\
\hline$\lambda$ & $\mathrm{m}^{2} \mathrm{~s}^{-3}$ & $\begin{array}{l}\text { export rate of TKE out of CL } \\
\text { other }\end{array}$ \\
\hline$a_{i}$ & $\ldots$ & weighting factor for $\mu_{i}$, \\
\hline$c_{B}$ & & Batchelor constant \\
\hline$c_{p}$ & $\mathrm{~J} \mathrm{~K}^{-1} \mathrm{~kg}^{-1}$ & $\begin{array}{l}\text { heat capacity of water at constant pressure } \\
\text { displacement vector, representing } \Pi\end{array}$ \\
\hline$g$ & $\mathrm{~m} \mathrm{~s}^{-2}$ & $\begin{array}{l}\text { gravitational acceleration } \\
\text { s. }\end{array}$ \\
\hline I & $\mathrm{K} \mathrm{m} \mathrm{s}^{-1}$ & kinematic radiation flux \\
\hline$I_{0}$ & $\mathrm{~K} \mathrm{~m} \mathrm{~s}^{-1}$ & surface radiation flux, $I_{0}=I(z=0)$ \\
\hline$k$ & cpm & wave number \\
\hline$N^{2}$ & $\mathrm{~s}^{-2}$ & stability, squared Brunt-Väisälä frequency \\
\hline$r_{m}$ & $\mathrm{~m} \mathrm{~s}^{-1}$ & meltwater inflow rate \\
\hline$S$ & psu & salinity \\
\hline$t$ & s & $\begin{array}{l}\text { time, relative to the first day of } \\
\text { measurements (April 19, 1999); } \\
\text { skipping the zero, April } 19 \text { is called day } 1 \\
\text { while April } 18 \text { is called day }-1\end{array}$ \\
\hline$T$ & ${ }^{\circ} \mathrm{C}$ & temperature \\
\hline$z$ & $\mathrm{~m}$ & $\begin{array}{l}\text { water depths with origin at } \\
\text { the ice/water interface }\end{array}$ \\
\hline$z_{\text {ref }}$ & $\mathrm{m}$ & arbitrary reference depth \\
\hline$\Delta t$ & $\mathrm{~s}$ & model time step \\
\hline$\Delta z$ & $\mathrm{~m}$ & model grid spacing \\
\hline$\Pi$ & & $\begin{array}{l}\text { permutation defining a numerical } \\
\text { stabilization procedure }\end{array}$ \\
\hline$\alpha$ & $\mathrm{K}^{-1}$ & thermal expansivity of water \\
\hline$\beta$ & $\mathrm{m} \mathrm{s}^{-2} \mathrm{~K}^{-1}$ & buoyancy parameter, $\beta=g \alpha$ \\
\hline$\delta$ & & model grid node index \\
\hline$\phi_{T}$ & $\mathrm{~K}^{2} \mathrm{cpm}^{-1}$ & Batchelor spectrum for temperature \\
\hline$\kappa_{S}$ & $\mathrm{~m}^{2} \mathrm{~s}^{-1}$ & molecular diffusivity of salt in water \\
\hline$\kappa_{T}$ & $\mathrm{~m}^{2} \mathrm{~s}^{-1}$ & molecular diffusivity of heat in water \\
\hline$\mu_{i}$ & $\mathrm{~m}^{-1}$ & extinction coefficient \\
\hline & $\mathrm{m}^{2} \mathrm{~s}^{-1}$ & kinematic viscosity of water \\
\hline$\rho$ & $\mathrm{kg} \mathrm{m}^{-3}$ & density of water \\
\hline$\tau$ & $\ldots$ & model temporal index \\
\hline$\xi$ & $\ldots$ & dimensionless depth \\
\hline & & superscripts, subscripts, overbars \\
\hline$u^{\text {ice }}$ & & bulk property of the ice \\
\hline$u^{*}$ & & variation of $u$ (different conditions etc.) \\
\hline$u_{0}$ & & at the ice/water interface, $u_{0}=u(z=0)$ \\
\hline $\bar{u}$ & & averaged in $\mathrm{CL}, \bar{u}=1 /\left(z_{2}-z_{1}\right) \int_{\mathrm{CL}} u(z) d z$ \\
\hline$\tilde{u}$ & & averaged in EL, $\tilde{u}=1 /\left(z_{3}-z_{2}\right) \int_{\mathrm{EL}} u(z) d z$ \\
\hline
\end{tabular}




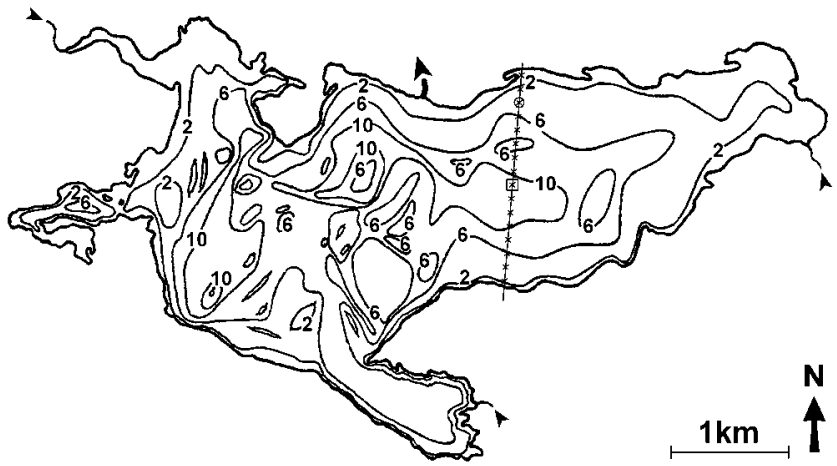

Figure 1. Bottom relief of Lake Vendyurskoe $\left(62^{\circ} 15^{\prime} \mathrm{N}\right.$, $\left.33^{\circ} 15^{\prime} \mathrm{E}\right)$. Holes for the CTD transects are marked with crosses along the straight line. The microstructure profiler and the meteorological station, as well as the thermistor string, are located at boxed cross. The submerged pyrradiometer is deployed at circled cross.

of about $1.5 \mathrm{~km}$, and a maximum depth of only $13.4 \mathrm{~m}$, it can be considered shallow. The lake's bottom relief is shown in Figure 1. According to Litinskaya and Polyakov [1975], Lake Vendyurskoe features a surface area of $10.4 \mathrm{~km}^{2}$ and a volume of $54.8 \mathrm{Mio} \mathrm{m}^{3}$ yielding a mean depth of $5.3 \mathrm{~m}$. Three small inflows and one outflow balance at an annual average flow rate of only $0.6 \mathrm{~m}^{3} \mathrm{~s}^{-1}$. The geological conditions of the drainage basin govern the lake's bulk salinity of about 0.02 psu [Malm et al., 1997a].

[7] A "SeaBird" temperature microstructure profiler was installed for a cable-constrained uprising profiling mode (Figure 2) based upon the setup by Kocsis et al. [1999]. To ensure profiling through the undisturbed TL, the device was deployed underneath a closed patch of ice. The profiler was rising at a speed of $6 \mathrm{~cm} \mathrm{~s}^{-1}$. To prevent damage of the probe, the profiler was halted $10 \mathrm{~cm}$ before the sensitive sensor tip would have touched the ice.

[8] A thermistor string of 10 self-contained temperaturelogging units was deployed close to the profiling site. The sensor's positioning was set up to particularly investigate the CL boundaries. Nearby, a meteorological station registered incoming and outgoing long- and short-wave radiation. Air temperature and relative humidity were also recorded. Further away, a submersible upward-looking pyrradiometer was positioned directly beneath the ice. A transect of 16 holes across the ice-covered lake served for regular CTD casts as indicated in Figure 1.

\subsection{Temperature Microstructure Method}

[9] Microscale temperature fluctuations have been utilized to unravel the physics of turbulence since the 1960s, when appropriate technology became available. For a brief history see the work of Gregg [1991]. Batchelor [1959] theoretically estimated the form of the temperature gradient spectrum for isotropic, homogenous turbulence. This spectrum was reformulated in a one-dimensional form and tested against laboratory experiments by Gibson and Schwartz [1963]. Since then, the comparison of model spectra and measured gradient spectra has been utilized to estimate the dissipation rate of TKE and of temperature variance [e.g.,
Gregg et al., 1973; Ivey and Imberger, 1991; Sander et al., 2000].

[10] We utilize an associated form of the single-sided, one-dimensional "Batchelor" spectrum $\phi_{T}$ for temperature

$$
\phi_{T}(k)=\frac{\chi}{2 \kappa_{T}} \frac{x^{3}}{k^{3}}\left(\frac{\mathrm{e}^{-x^{2}}}{x}-\left(\pi^{1 / 2}-2 \int_{0}^{x} \mathrm{e}^{-y^{2}} d y\right)\right),
$$

with

$$
x=k\left(c_{B} \kappa_{T}\right)^{1 / 2}\left(\frac{\nu}{\varepsilon}\right)^{1 / 4}
$$

where $k$ is the one-dimensional wave number, $c_{B}=3.4$ [Gibson and Schwartz, 1963; Grant et al., 1968; Dillon and Caldwell, 1980; Oakey, 1982] is the "Batchelor" constant, $\nu$ is the kinematic viscosity of water, and $\kappa_{T}$ is the molecular thermal diffusivity in water. Fitting $\phi_{T}$ onto spectra of observed temperature data provides estimates of the dissipation rates $\varepsilon$ of TKE and $\chi$ of temperature variance as fit parameters (details in the work of Simon [1997] and Gloor [1995]). Thanks to the excellent quality of the obtained data, reliable estimations of $\varepsilon$ down to $10^{-11} \mathrm{~m}^{2} \mathrm{~s}^{-3}$ were possible. This very low detection limit was necessary to resolve the dynamics of radiatively driven convection under the given circumstances.

[11] To ensure that the measurements were unaffected by profiling-induced eddies, the deployment of the profiler had to meet a certain downtime allowing artificial turbulence to decay (dissipatively and/or advectively) between successive profiles. As the horizontal advection velocities could not be measured, turbulence decay tests were carried out to define the minimum downtime. Therefore the profiler was dragged downward at high speed to induce artificial eddies, halted, and then measured the gradual decay of the self-generated turbulence. A minimum downtime of 1.5 hours was considered appropriate after several tests during periods when the lake featured no detectable natural turbulence $\left(\varepsilon<10^{-11} \mathrm{~m}^{2} \mathrm{~s}^{-3}\right)$. However, to begin cautiously, the first day profiles were taken only every

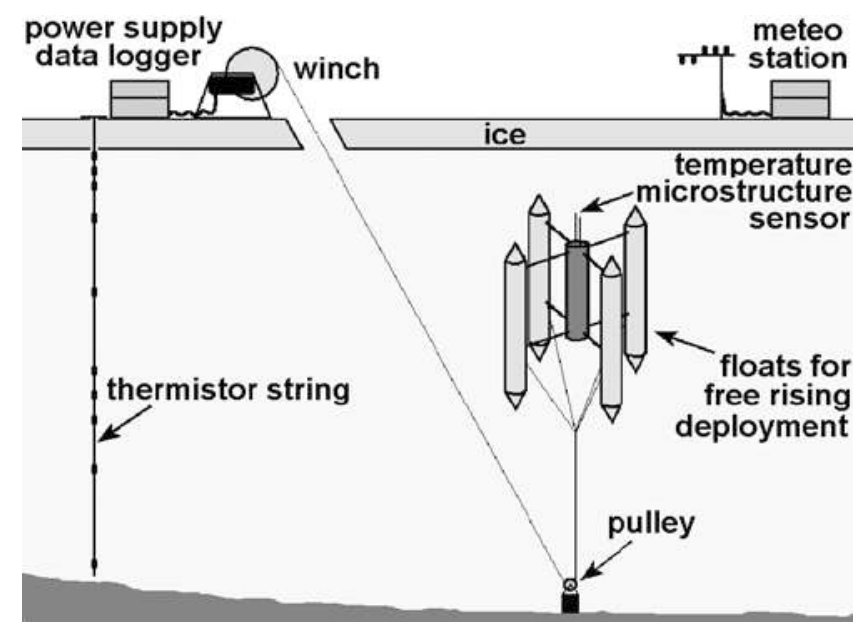

Figure 2. Experimental setup and deployment mode of the temperature microstructure profiler. 
6 hours, the second day every 3 hours, and afterward every 1.5 hours.

\subsection{Model: Motivation and Setup}

[12] A one-dimensional model has been developed to predict the temperature and salinity stratification by means of the surface radiation flux and the inflow rate of water from melted ice. It is based upon the transfer equations for heat and salinity and includes a procedure to neutralize unstable stratification.

[13] The motivation for the model development originates from the lack of high-resolution salinity data synchronous to the temperature microstructure profiles. As the temperature of the $\mathrm{CL}$ approaches the temperature of maximum density, the salinity is of increasing importance for the density stratification in spite of its low absolute magnitude of about $S=0.02 \mathrm{psu}$ in the CL. The stratification dynamics is governed by the complex interplay between temperature and salinity. Hence besides indirect conclusions and speculations, the model provides the only way to characterize this interplay, provided it performs consistently. Fortunately, besides the consecutive highresolution temperature profiles, low-resolution salinity data are available from a nearby site ( $20 \mathrm{~m}$ away) on a regular basis providing a reference for the modeled high-resolution salinity profiles.

[14] As a second motivation, a model allows testing different parameterizations of the vertical absorption pattern of radiation, where an appropriate parameterization is crucial for the quantification of the production of buoyancy. A thin layer between the CL and the ice is primarily governed by the absorption of radiation and by molecular diffusion. As the latter mechanism is easy to model, the former can be tuned to match the observed near-ice stratification. Thus the model helps to optimize the parameterization of the vertical absorption pattern of radiation.

[15] Without advection and inflow processes, temperature $T$ and salinity $S$ evolve in the water column according to

$$
\begin{gathered}
\frac{\partial T}{\partial t}=-\frac{\partial I}{\partial z}+\kappa_{T} \frac{\partial^{2} T}{\partial z^{2}}, \\
\frac{\partial S}{\partial t}=\kappa_{S} \frac{\partial^{2} S}{\partial z^{2}},
\end{gathered}
$$

where $I$ is the kinematic flux of solar radiation (radiative heat flux divided by density $\rho$ of water and the specific heat $c_{p}$ of water at constant pressure), and $\kappa_{S}$ is the molecular diffusivity of salt in water. The convective procedure to deal with unstable stratification leads to a coupled alteration of the temperature and salinity stratification and is treated solely numerically. The same applies for the inflow of water from melting ice.

[16] The model is initialized by the input of the temperature and salinity stratification. Per time step, these profiles are subject to the following procedures: accumulation of absorbed radiation, the inflow of meltwater, neutralization of density instabilities, and molecular diffusion. Contrary to common adjustment procedures (complete mixing proce- dures), we employ a modified adiabatic, nonmixing reaction to density instabilities (Appendix A). Three different versions of this procedure are discussed in section 4.3.

[17] The numerical procedures operate centered in space and forward in time. The model grid is defined relative to the ice/water interface with a vertical resolution of $0.5 \mathrm{~mm}$ as given by the temperature microstructure profiles. The time step has to result in typical convective displacement lengths that are significantly greater than the grid spacing, and significantly smaller than the CL depth. A time step of $150 \mathrm{~s}$ fulfills these requirements. To ensure the numerical stability of the molecular diffusion process, a time-splitting procedure was introduced, altering the stratification at a 1000-fold smaller timescale.

[18] A detailed description of the model's numerics is given in Appendix A, and a discussion of its performance follows in section 4.3.

\section{Driving Forces}

\subsection{The Solar Radiation Flux}

[19] Besides the buoyancy parameter $\beta$ ( $\beta=g \alpha$, where $g$ is the gravitational acceleration and $\alpha$ is the thermal expansivity of water), the solar radiation flux $I$ is the most important property for scaling radiatively driven turbulence. The surface radiation flux $I_{0}$ was measured directly beneath the ice/water interface using a submergible pyrradiometer. However, these measurements are only representative of the specific local conditions of the ice cover, underneath which the pyrradiometer was deployed. Considering the horizontal inhomogeneities in the opacity of the ice cover, a heat-budget based calibration seems essential to represent the conditions at the profiling site. Therefore the change rate of the total heat content of the water column above the stably stratified quiescent layer $(\mathrm{QL})$ is estimated, and then corrected by the heat fluxes into the ice and out of the QL, assuming quasi-stationary molecular heat flux at the interfaces. Because the budget domain is defined relative to the ice/water interface, an additional pseudo heat flux is to be taken into account: the water column is being shifted downward by meltwater inflow, where heat is only virtually lost by that part of the water column that in turn leaves the budget domain. Both the thermistor string data as well as the temperature microstructure data were used as such to determine the surface radiation flux (Figure 3 ). Some temporal averaging was necessary to filter the temporal fine structure of the diurnal cycle caused by advection of horizontal inhomogeneities of the temperature distribution.

[20] The two independent heat budget-based estimations of the surface radiation flux match well, while they exceed the measured flux by typically $50 \%$. The microstructure profiler as well as the thermistor string was deployed under a relatively transparent area of the ice cover, contrary to the submerged pyrradiometer. Apart from the naturally occurring patchy pattern of the ice cover, heat from the equipment and walking on the slush at the profiling site may additionally account for the discrepancy. We use the measured surface radiation flux multiplied by a factor of 1.50 for scaling and modeling purposes in the following as the temporal dynamics is better represented by the measurements, while the specific local amplitude of 


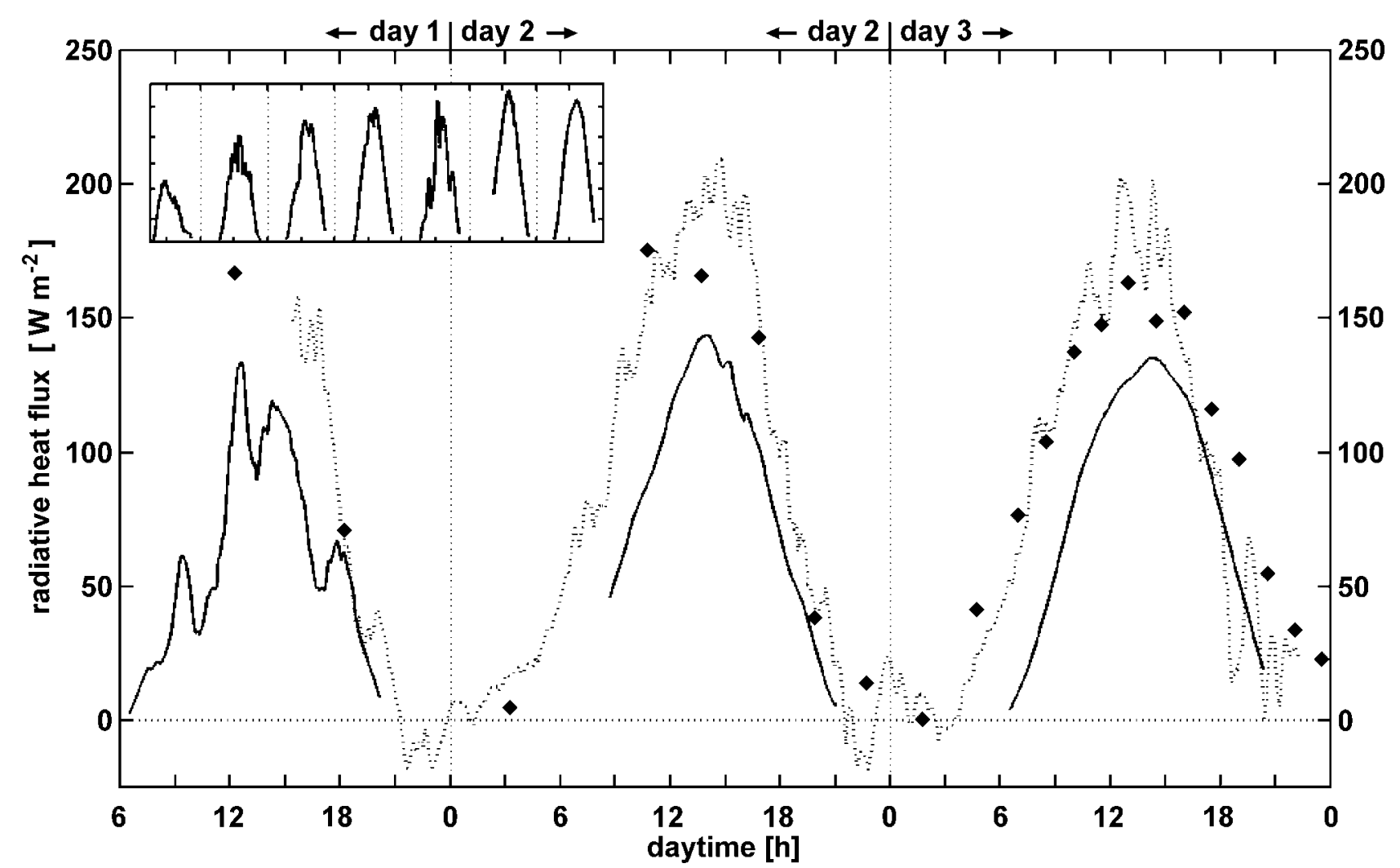

Figure 3. Radiative heat flux $\rho c_{p} I_{0}$ according to different estimation methods during the first 3 days of measurements: direct measurements (solid line); heat budget derived estimation on the basis of thermistor string data, running mean filtered ( \pm 1 hour) (dotted line); and heat budget derived estimation on the basis of microstructure CTD data, running mean filtered ( \pm 2 profiles) (solid diamonds). The inset displays the same measurements of the radiative heat flux but includes the preceding 4 days (vertical axis limited from 0 to $150 \mathrm{~W} \mathrm{~m}^{-2}$ ).

the surface radiation flux should rather fit the heat-budget based estimations.

[21] The accumulation of radiated heat in the water is proportional to the vertical divergence of the radiation flux $I(z, t)$

$$
\frac{\partial T}{\partial t}(z, t)=-\frac{\partial I}{\partial z} \cong I_{0}(t) \sum_{i} \mu_{i} a_{i} \mathrm{e}^{-\mu_{i} z}
$$

where the exponential multiband parameterization on the right-hand side is commonly used to describe the solar radiation flux in water. Here the cumulative absorption of radiation in the TL between the ice and the $\mathrm{CL}$ is of particular interest (section 5.1). The coefficients $\mu_{i}$ and $a_{i}$ were estimated by matching model predictions to the observed temperature stratification in the TL (Figure 4). This fitting method is expected to reveal an appropriate parameterization, as the physics close to the ice/water interface should be appropriately represented by our model: only absorption of radiation, molecular diffusion, and the inflow of water from melted ice are relevant processes that govern the near-ice stratification. Unfortunately, though, the top $10 \mathrm{~cm}$ of the microstructure profiles are not available for reasons of cautious profiling, as previously mentioned.

[22] In Figure 4, observational data of the near-ice stratification and model predictions according to two types of parameterizations of $I(z, t)$ are shown. A two-band parameterization does not allow predictions that match observational data accurately in the vicinity of the ice/water interface, as also demonstrated by Malm et al. [1996, Figure 27]. The larger of the two extinction coefficients predominately governs the predicted stratification in the near-ice region, but the tilt rather than the curvature of the temperature profiles can be influenced. Much better predictions are obtained by using a one-band parameterization and by coupling the extinction coefficient to the salinity (Figure 4),

$$
\mu(S)=\mu^{\text {ice }}+\left(\bar{\mu}-\mu^{\text {ice }}\right) \frac{S-S^{\text {ice }}}{\bar{S}-S^{\text {ice }}}
$$

where $S^{\text {ice }}=0.002 \mathrm{psu}$ is the salinity of the meltwater, and $\bar{S}=0.02$ psu is the bulk salinity of the CL. This coupling was considered, assuming salinity to trace the diffusion of the bulk turbidity into the clearer water at the ice/water interface, which is being actively diluted by the inflow of meltwater. The fitting priorities were set to optimize the match between model predictions and the curvature at the top end of the microstructure profiles, as the performance of the model is most accurate where the TL is undisturbed by penetrative entrainment out of the CL. The best predictions were established with $\mu^{\text {ice }}=0.5 \mathrm{~m}^{-1}$ and $\bar{\mu}=3.5 \mathrm{~m}^{-1}$ for days 1 and 2 , and a similar set $\mu^{\text {ice }}=0.4 \mathrm{~m}^{-1}$ and $\bar{\mu}=2.7 \mathrm{~m}^{-1}$ for 

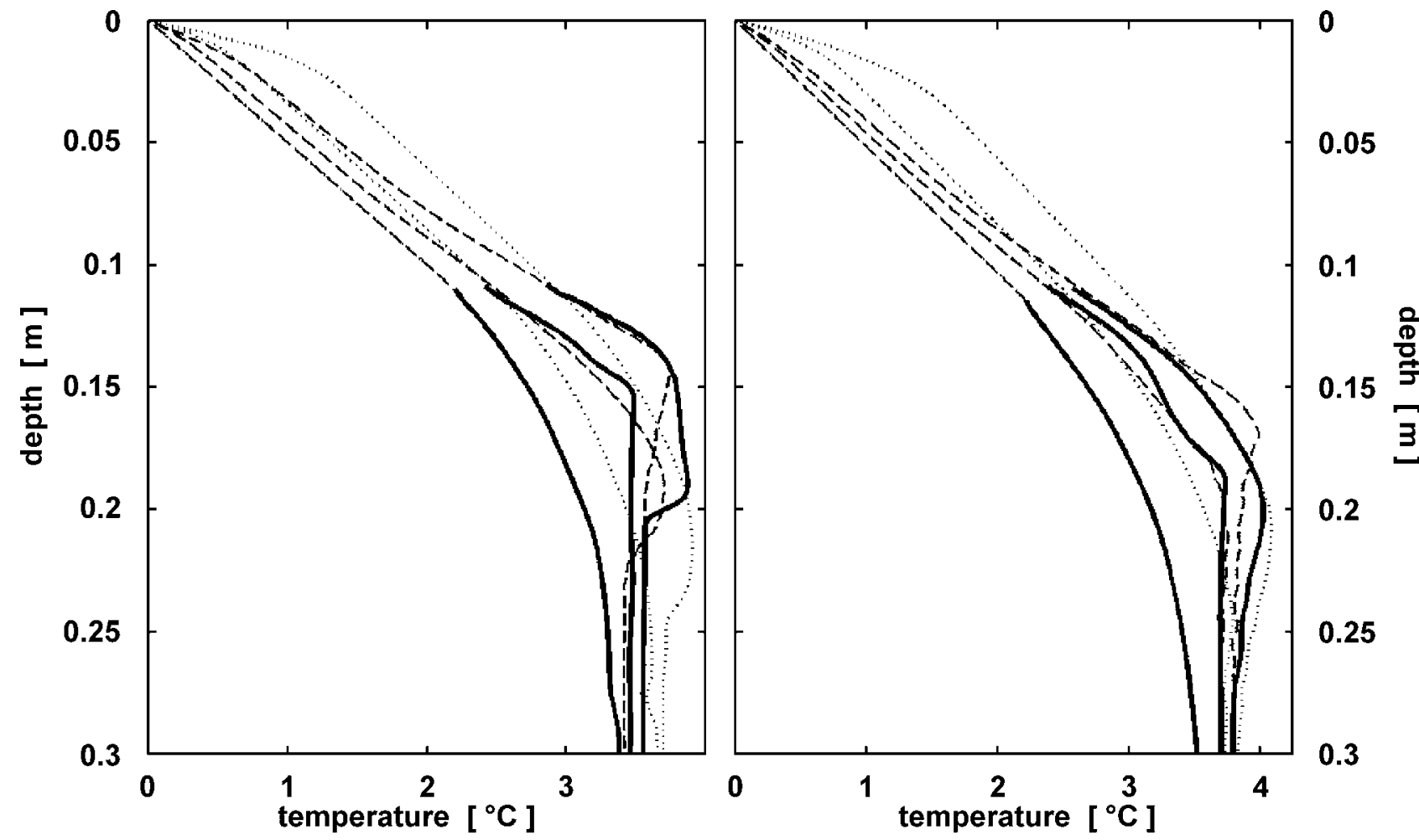

Figure 4. Observations and model predictions of the near-ice stratification on (left) day 2 and on (right) day 3: observations (thick solid lines); two-band parameterization according to equation (3) with $a_{1}=0.8$, $\mu_{1}=0.7 \mathrm{~m}^{-1}, a_{2}=0.2$, and $\mu_{2}=2.0 \mathrm{~m}^{-1}$ (dotted lines); one-band parameterization according to equations (3) and (4) with coefficients as given in the text (dashed lines). Left-hand panel for day 2, from left to right: starting profile at 0915 hours, comparing profiles at 1215 and 1515 hours. Right-hand panel for day 3 , from left to right: starting profile at 0745 hours, comparing profiles at 1215 and 1645 hours. Model setup according to run $\Pi+\Pi^{*}+\Pi^{* *}$ (section 4.3 and Appendix A).

days 3 and 4, respectively. We use equation (4) in (3) with $a_{1}=1$ in the following to parameterize the radiation flux $I(z, t)$ with the given sets of coefficients.

\subsection{The Melting Process}

[23] The melting progress of the ice cover determines its permeability to light and therefore governs the intensity of convection. Moreover, because melted ice and snow partially enters the water column, its inflow rate is of significance for the TL dynamics. The meltwater particularly affects the absorption of radiation above the CL, and is therefore directly linked to the availability of potential energy for the generation of TKE. Hence it is important to thoroughly consider the inflow rate of water from melting ice.

[24] Several direct measurements of the cover thickness reveal an average melting rate of $2.5 \mathrm{~cm} \mathrm{~d}^{-1}$ for the period from days -8 to +6 with a trend of increasing up to $3.3 \mathrm{~cm}$ $\mathrm{d}^{-1}$ for days +2 to day +6 , where day 1 refers to 19 April 1999 (Table 1). The cover consists of an ice body and an overlaying snow layer, which degrades to wet slush with its progressive thermal destruction. This degrading process took place primarily during the week before the measurements began when a snow layer of $5 \mathrm{~cm}$ melted down to $1.5 \mathrm{~cm}$ of wet slush. Consistently, the surface heat flux strongly increased day by day during that week, but only moderately afterward (inset of Figure 3).
[25] To estimate the diurnal melting dynamics of the ice cover, we assembled a heat budget (Figure 5). As the remaining slush seemed to have roughly become as transparent as the ice until day 1 , we do not separate the ice cover into sublayers for the heat budget. Melting in the interior, at the top interface, and at the bottom interface of the ice/slush cover is separately considered. The average air temperature was found well above $0^{\circ} \mathrm{C}$ during the measurements and at least during 1 week in advance. Moreover, internal melting of the ice has been observed to lead to the formation of vertical channels in the ice. Therefore we may roughly assume that the complete ice body was already heated to $0^{\circ} \mathrm{C}$ by day 1 . Hence the absorption of short-wave radiation within the cover contributed to internal melting (Figure 5, dotted line). Despite the positive air temperatures, the slush cover emitted more long-wave radiation than it absorbed from atmospheric radiation, accounted for by the different emissivities of the atmosphere and the slush (Figure 5, dashed-dotted line). Therefore some freezing is expected to have occurred during the nights. As for the melting due to sensible and latent heat flux at the air/ice interface, the lack of wind data and information about the roughness length of the slush allowed for rough estimations of the respective contribution only. According to bulk formulae by Price and Dunne [1976], sensible and latent heat accounted for $0.6 \mathrm{~cm} \mathrm{~d}^{-1}$ at the most (Figure 5, dashed curve), estimating a wind speed $u_{1}$ of $1 \mathrm{~m} \mathrm{~s}^{-1}$ maximum and a roughness length of $1 \mathrm{~cm}$ 


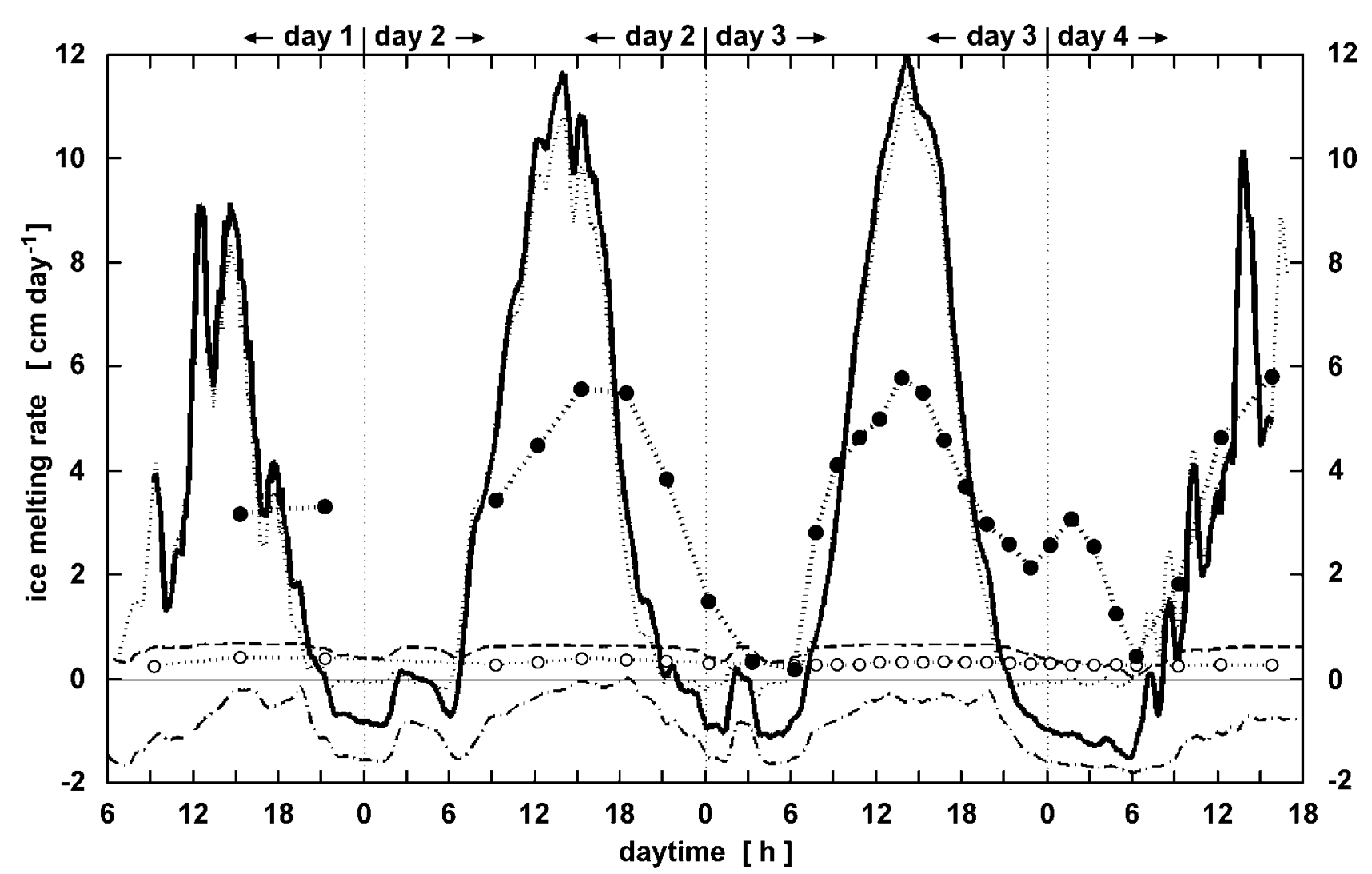

Figure 5. Melting rate of the ice cover, its contributing terms, and thickness decrease rate of ice cover: total melting rate (thick solid line); contribution by absorption of short-wave solar radiation (incoming reflected - penetrated) (dotted line); contribution by long-wave radiation balance (absorbed - emitted) (dash-dotted line); maximum contribution by latent and sensible heat flux (dashed line); contribution by heat flux from water (open circles on dotted line); and decrease rate of ice-cover thickness (solid circles on thick dotted line).

maximum. Because we presume the ice cover featured a homogeneous temperature of $0^{\circ} \mathrm{C}$, the melting at the ice/ water interface is determined by the heat flux from the water to the ice only. As measurements of the temperature gradient directly below the interface are not available, the gradient was estimated by linear extrapolation of the data to $T(z=0)=0$. However, with an average contribution of $0.31 \mathrm{~cm} \mathrm{~d}^{-1}$ only, this inaccuracy seems insignificant (Figure 5, open circles upon dotted lines). The sum of the discussed contributions gives the total rate $r_{m}$ (hereafter referred to as meltwater inflow rate, Figure 5 , thick solid line). Averaged over the period from days 1 to 3 , we get a rate of 2.9 and of $3.4 \mathrm{~cm} \mathrm{~d}^{-1}$ averaged over the two cloudless days 2 and 3 . These findings match the measured values very well. The budget reveals that internal melting due to the absorption of short-wave radiation is the dominant mechanism. Because the latent heat flux seems negligible and no meltwater on the slush was observed, we may assume that most of the melted ice cover flowed into the water column. Further details can be found in Appendix B.

\section{Observations of the Daytime Stratification Dynamics}

[26] The density stratification of the lake is governed by the complex interplay of temperature and salinity distribu- tion. Thus it is unfortunate that we lack high-resolution salinity data, simultaneous to the microstructure profiles. Available are, however, CTD profiles from a nearby site with a vertical resolution of $5 \mathrm{~cm}$, which is not sufficient to observe the TL daytime dynamics in full detail.

[27] The model was developed to compensate for this deficiency. By modeling the observed stratification dynamics, an approximation of the lacking salinity data can be generated. However, most of the stratification dynamics can be analyzed by means of the measured data only. To simplify the further discussion, the water column is subdivided into distinct layers by defining interfaces at $z_{0}$ to $z_{3}$ (Figure 6). The CL is enclosed by two layers, both of which feature a stabilizing salinity gradient but a destabilizing temperature gradient (in the TL only temporarily). However, the stabilizing salinity gradient always dominates the stratification. At the boundaries of the CL, the complex dynamics are governed by interactions between the convective flow and the ambient stratification. To assess these interactions, the following analysis of the stratification cycle focuses on the local dynamics around these boundaries.

[28] In the first panel of Figure 7, successive microstructure profiles demonstrate the daytime dynamics of the temperature stratification, as observed during the final period of strong radiatively driven convection (day 3). 


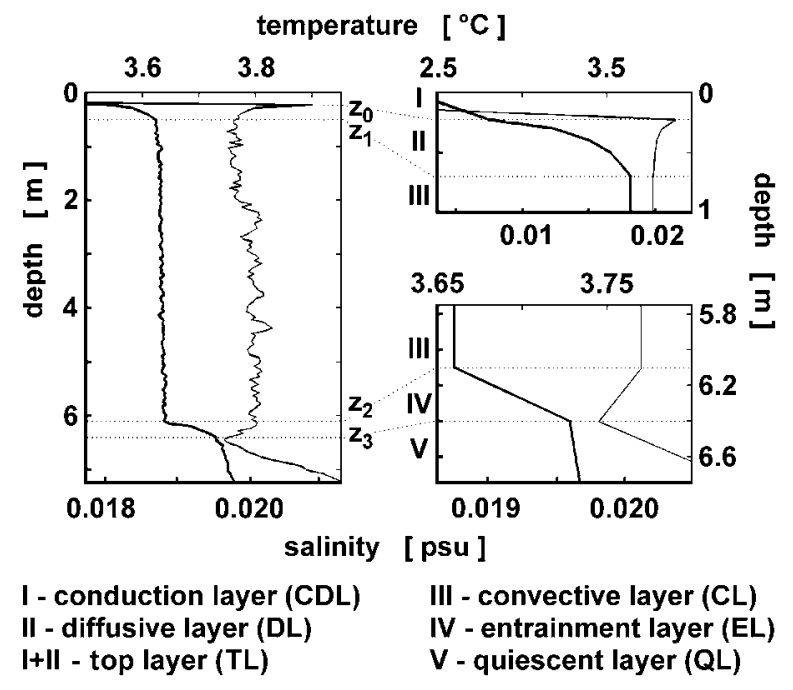

Figure 6. Typical afternoon profiles of salinity (thick lines, left and bottom axes) and of temperature (thin lines, right and top axes). Left-hand panel is observational data, two right-hand panels are schematic enlargements. Layers and their interfaces are defined as indicated by thin dotted lines. The names, acronyms, and letters are defined as further reference. Notice that the temperature maximum at $z_{0}$ occurs only between noon and late evening.

Note that in the vicinity of the temperature of maximum density, temperature fine structures may be comparatively large due to the small buoyancy parameter $\beta$. Because of the extraordinarily fast melting progress of the ice cover, the water body was still at low temperatures when the measurements began, while the penetration by the solar radiation was already intense. The mixed layer warmed rapidly at $0.2 \mathrm{~K} \mathrm{~d}^{-1}$, leading to a short but intense convection period. The measurements started only 4 days before the bulk temperature $\bar{T}$ of the CL reached the temperature of maximum density.

[29] In the following, we particularly focus on the daytime dynamics as observed during the period from days 1 to 3 . As $\bar{T}$ is below the temperature of maximum density, the accumulation of radiation causes the generation of density instabilities in the quasi-mixed $\mathrm{CL}(d I / d z<0$, equation (3)). The equilibration of these instabilities results in a coupled transport of salinity and temperature through the $\mathrm{CL}$.

\subsection{Entrainment Layer Dynamics}

[30] Due to the stratification setup around the upper boundary $z_{1}$ of the CL, water parcels that are convectively transported downward feature a lower salinity and, during most of the day, a higher temperature, compared to the corresponding bulk values of the CL. Upon penetrating the interface to the entrainment layer (EL) below, these water parcels cause the interface at $z_{2}$ to lower, while the stratification in the upper EL remains quasistationary. Instead, the lower boundary $z_{3}$ of the EL deepens synchronously to $z_{2}$ (Figure 7). This forced deepening of $z_{3}$ suggests that the entrainment penetrates well down to the interface between EL and the QL below.

[31] The quasiconservation of the bulk EL stratification implies that the CL and the EL deepen proportionally to
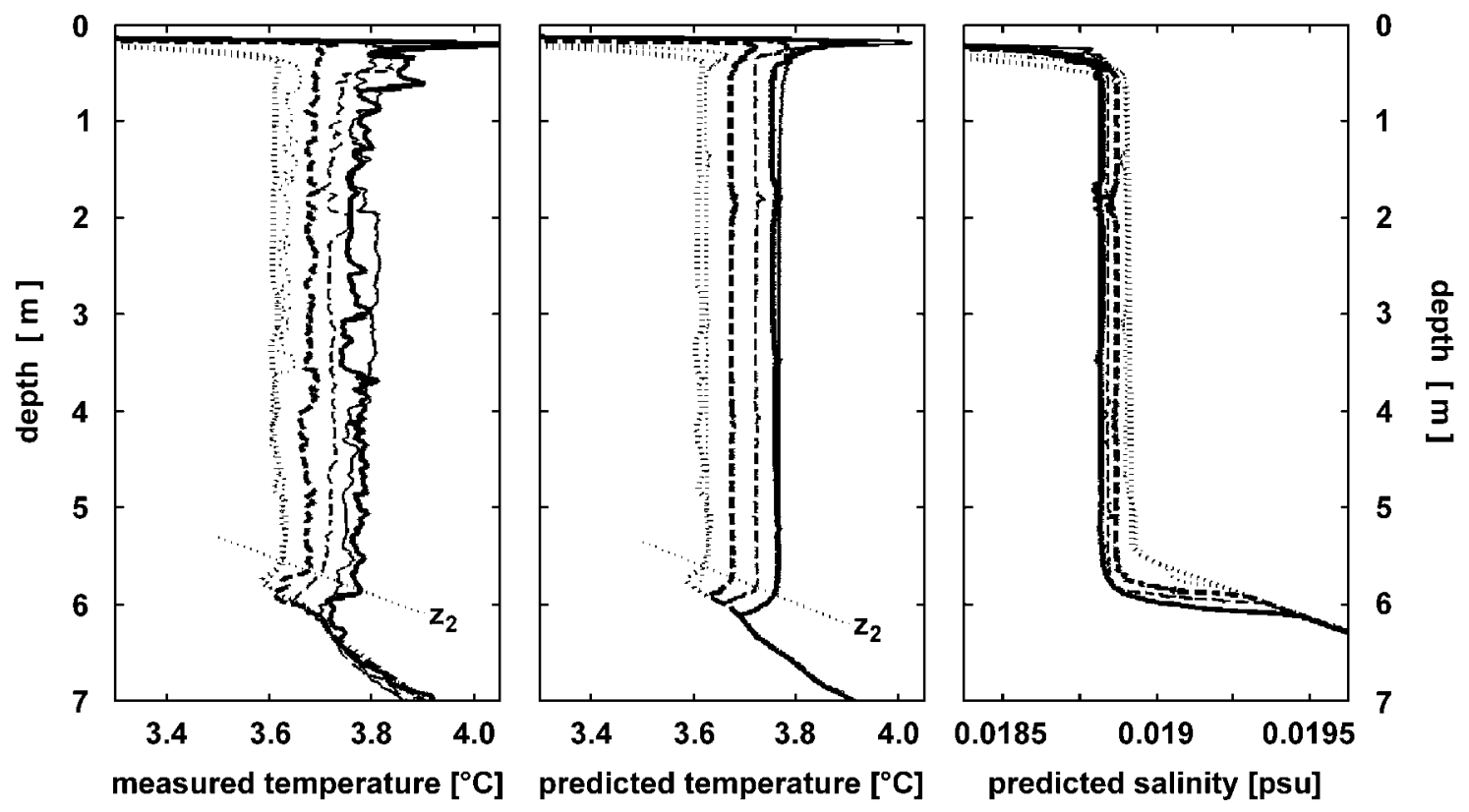

Figure 7. Measured and predicted daytime stratification on day 3. Initialization of the model with the observed temperature stratification on day 3 at 0745 hours, and closest available observed salinity stratification of day 2 at 1945 hours. For temperature data from left to right, and for salinity data from right to left, respectively: 0745 hours (thick dotted line), 0915 hours (thin dotted line), 1215 hours (thick dashed line), 1515 hours (thin dashed line), 1645 hours (thick solid line), 1815 hours (thin solid line). The base of the convective layer $z_{2}$ is indicated by a thin dotted auxiliary line. Several measured salinity profiles are available only for the afternoon and match the dynamics of the predicted stratification well. Model setup according to $\Pi+\Pi^{*}+\Pi^{* *}$ (section 4.3 and Appendix A). 

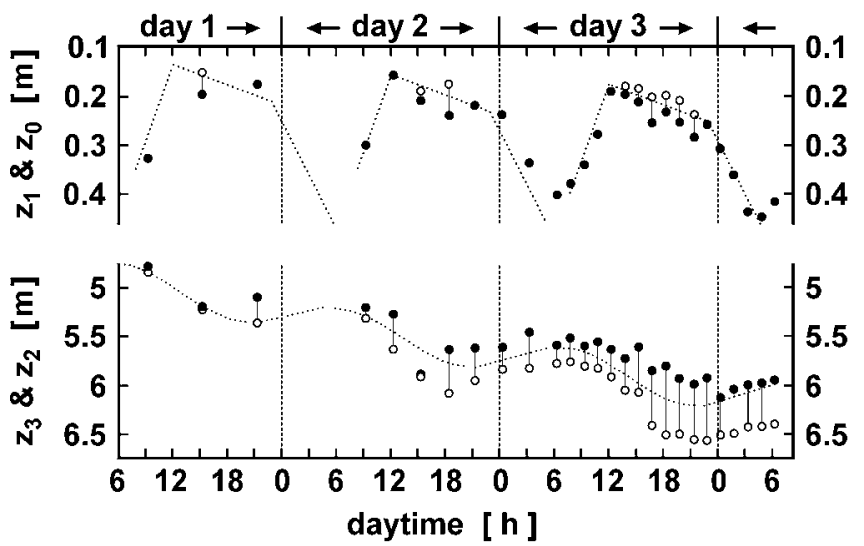

Figure 8. Vertical position of the layer interface $z_{0}$ (open circles, top panel), $z_{1}$ (solid circles, top panel), $z_{2}$ (solid circles, bottom panel), and $z_{3}$ (open circles, bottom panel) as evaluated from consecutive temperature microstructure profiles (for layer definitions see Figure 6). The thin dotted lines schematically depict the diurnal interface dynamics (see text for further explanations).

$d \bar{T} / d t$, and hence proportionally to $I_{0}(t)$. Looking at the daytime dynamics of $z_{2}$ and $z_{3}$, we find the correlation confirmed (Figure 8, bottom panel). A prerequisite for the development of an EL featuring a destabilizing temper- ature gradient is the convective transport of low-salinity water from the TL (Figure 6) downward through the CL. It allows building up a layer beneath the CL with an enhanced salinity gradient, capable of overcompensating the destabilizing temperature stratification. Hence such stratification setup in the EL may only evolve if a significant positive salinity gradient exists above the CL, which means that the ice needs to feature a lower salinity than the bulk CL salinity. As the salinity gradient can only overcompensate the destabilizing temperature stratification if $\beta$ is sufficiently small, this special stratification regime of the EL is expected to occur only during the final period of strong convection. An extrapolation of the EL thickness before day 1 would support this expectation (Figure 8, bottom panel).

\subsection{Top Layer Dynamics}

[32] The diffusive layer (DL) between the conduction layer (CDL) and the CL may be considered as the symmetric equivalent to the EL: both the DL and EL feature the same stratification setup, where salinity is stabilizing and temperature is destabilizing the water column, and both layers are subject to entrainment fluxes from the CL. But two differences are prominent. First, the DL is directly driven by the absorption of radiation, and is not only subject to the dynamics of its neighboring layers. Second, molecular diffusion destroys the DL every night, so that its
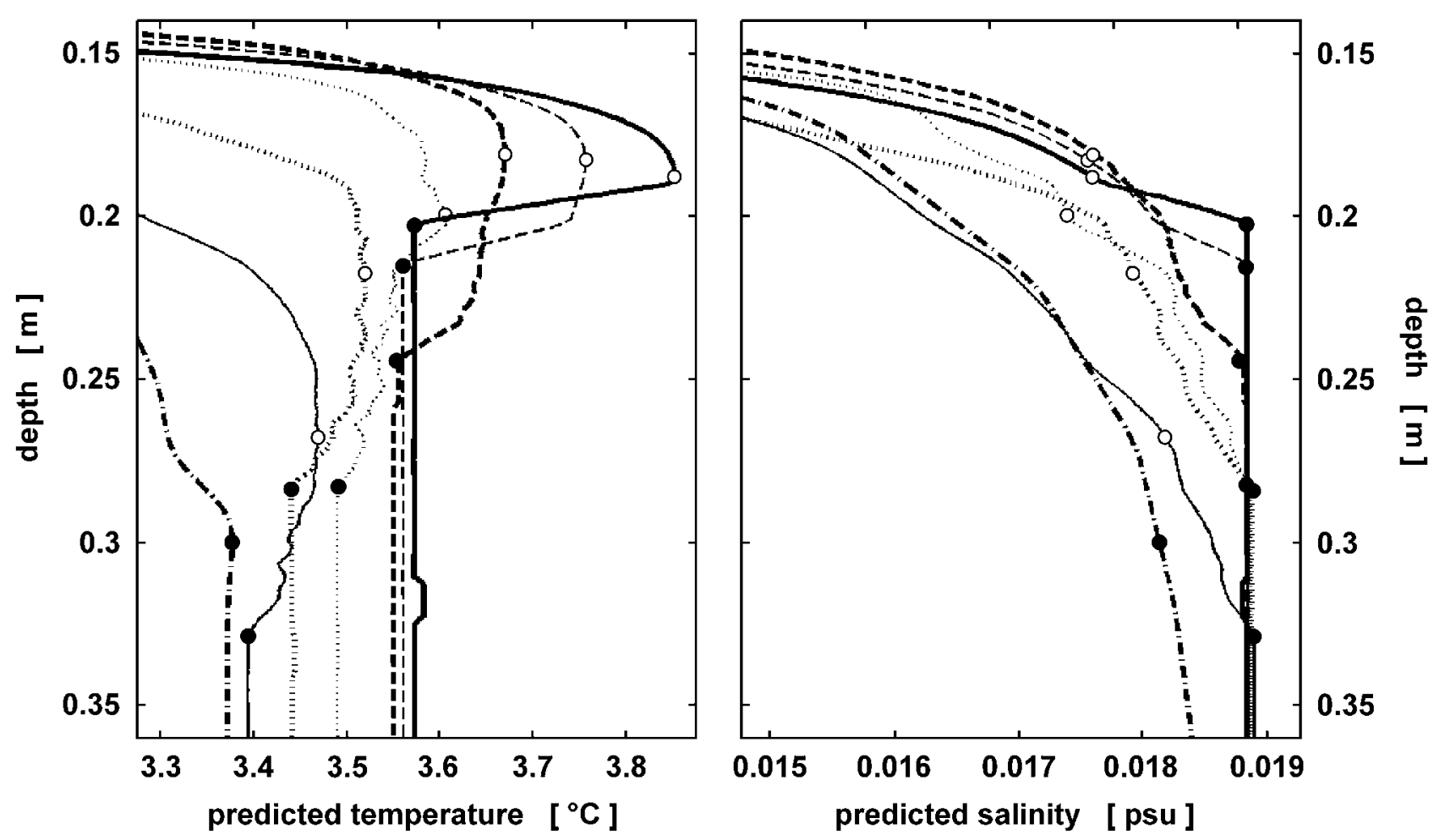

Figure 9. Model predictions of the stratification dynamics in the top layer. Initialization of the model with observed stratification on day 2 at 0915 hours. From left to right in respective time intervals of 1 hour, that is: 0915 hours (thick dash-dotted line), 1015 hours (thin solid line), 1115 hours (thick dotted line), 1215 hours (thin dotted line), 1315 hours (thick dashed line), 1415 hours (thin dashed line), 1515 hours (thick solid line). Open circles indicate position of interface $z_{0}$, solid circles indicate position of interface $z_{1}$, respectively. Model setup according to run $\Pi+\Pi^{*}+\Pi^{* *}$ (section 4.3 and Appendix A). 
measured temperature $\left[{ }^{\circ} \mathrm{C}\right]$
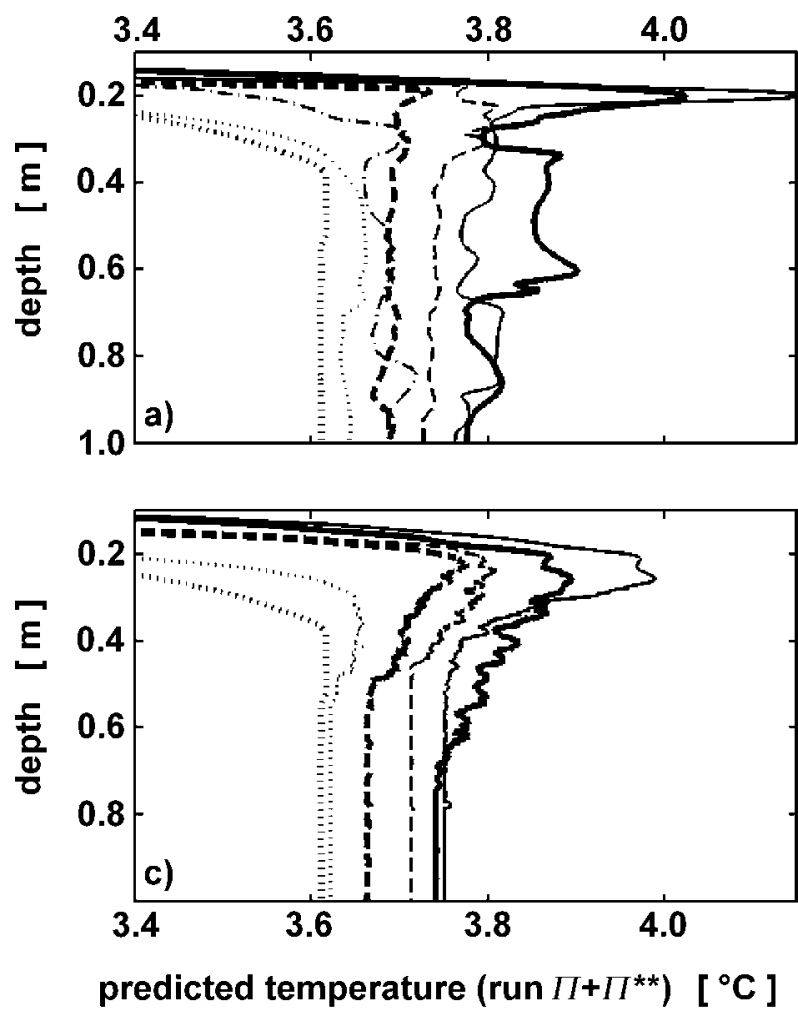

predicted temperature $(\operatorname{run} \Pi) \quad\left[{ }^{\circ} \mathrm{C}\right]$
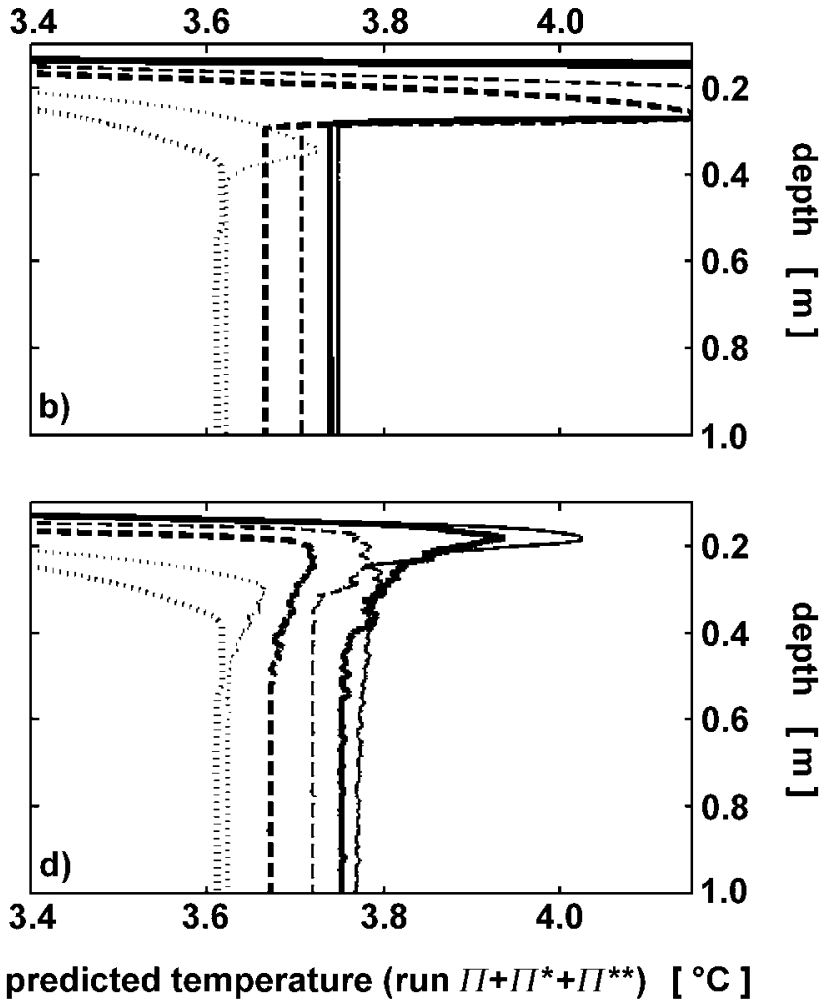

Figure 10. (a) Measured and $(b-d)$ predicted daytime temperature stratification with different model setups. Initialization of the model with observed temperature stratification on day 3 at 0745 hours, and closest available observed salinity stratification on day 2 at 1945 hours. From left to right: 0745 hours (thick dotted line), 0915 hours (thin dotted line), 1045 hours only observations (thin dotted line), 1215 hours (thick dashed line), 1515 hours (thin dashed line), 1645 hours (thick solid line), 1815 hours (thin solid line). Model setup (Figure $10 \mathrm{~b}$ ) according to run $\Pi$, (Figure $10 \mathrm{c}$ ) according to run $\Pi+\Pi^{* *}$, and (Figure 10d) according to run $\Pi+\Pi^{*}+\Pi^{* *}$ (section 4.3 and Appendix A).

dynamics follow a diurnal evolutionary cycle (top panel of Figure 8). Our model was employed to assess the complex daytime dynamics of the TL (Figure 9). Due to the stratification setup around the lower boundary $z_{2}$ of the CL, water parcels that are convectively transported upward feature a higher salinity and a lower temperature, compared to the corresponding bulk values of the CL. Two daytime periods can be distinguished.

[33] (1) In the morning no DL is developed yet and subsequently $z_{0}=z_{1}$ (Figure 8 ). The accumulation of radiation reduces the still positive temperature gradient at the base of the TL. Water parcels that convectively transport cold water upward seem to be incapable of compensating for this trend, as observations indicate. Instead, the high salinity of these updraft parcels sharpens the salinity gradient at the base of the TL, allowing the temperature gradient to become negative without inducing density instabilities. Thus the TL separates into a broad DL with a CDL above (Figure 9, first panel, left two curves). From then on, entraining water parcels from the CL tend to sharpen the DL via both the temperature and the salinity gradient. In the further course of the morning, the TL is compressed due to the convective transport of salinity upward, as the interface $z_{1}$ follows the sharp salinity bend (solid circles in Figure 9).

[34] (2) The transition to the afternoon dynamics of the TL seems to be induced by reaching a flux balance between molecular diffusion and heat accumulation, establishing a critical stability of the stratification in the CDL. When in the afternoon the surface radiation flux declines while the meltwater inflow rate is on its peak level, then the meltwater pushes the TL slowly back downward (Figure 8, top panel; Figure 9, right most three curves).

\subsection{Model Predictions of the Daytime Stratification Dynamics}

[35] In Figure 7, the observed daytime stratification is compared to model results. The model was initialized with the first temperature profile shown. For the initialization of the salinity stratification, the closest available profile (1945 hours the day before, closest CTD sampling site) was remapped onto the common high-resolution depth axis. Model results were output for the same times as the observational data.

[36] Despite its simple setup, the model can cope with the complex dynamics, and the observed and predicted profile 
structures compare well. To identify key mechanisms and their relevance for the stratification dynamics, the model was deployed for several runs, using the different available procedures modeling the system's reaction to density instabilities (Figure 10, for details see Appendix A). Since essentially only the prediction of the TL dynamics is sensitive to the model tuning, we concentrate on this layer in the following:

\subsubsection{Run $\Pi$}

[37] In a first step, a stable stratification was enforced after each time step by employing an adiabatic, nonmixing stabilizing procedure (Appendix A, П). With this simplest mode, generally, too much heat is accumulated in the TL (Figures 10a and 10b). Accordingly, not enough heat is left to fully reproduce the observed warming of the CL. Shortly after the model initialization, the predicted temperature stratification displays a prominent temperature maximum at $z_{0}$, contrary to observations.

\subsubsection{Run $\Pi+\Pi * *$}

[38] To improve the predictions, it turned out most effective to additionally allow penetrative flow (Appendix $\left.\mathrm{A}, \Pi^{* *}\right)$, as it enables the important entrainment of salinity into the TL (section 4.2). The model predictions now display a more appropriate amount of heat being accumulated in the TL (Figures 10a and 10c). As water from the CL penetrates into the TL, in turn warm water from the TL is transported downward, thus constituting an effective mechanism to restrict the growth of the temperature maximum at $z_{0}$. The fast development of a prominent temperature peak at $z_{0}$ seems to be prevented, but also later when such a peak is actually observed. Moreover, this model run results in water parcels from the CL penetrating unrealistically far into the TL, because their convective displacement speeds are not limited by the procedures used so far (see change of CDL stratification from run $\Pi$ to run $\Pi+\Pi^{* *}$, Figures $10 \mathrm{a}$ and 10c).

\subsubsection{Run $\Pi+\Pi *+\Pi * *$}

[39] Introducing a limitation of the convective displacement speed indeed results in a better match between observations and model predictions (Appendix A, $\Pi^{*}$; Figures $10 \mathrm{a}$ and $10 \mathrm{~d}$ ). The penetration of water parcels far into the TL is inhibited, establishing an appropriate prediction of the CDL stratification. Moreover, coupling the displacement speed limit to the surface radiation flux leads to another enhancement: the temperature maximum at $z_{0}$ substantially sharpens only in late afternoon, when the already weaker entrainment allows the DL to regain a greater stability. Especially the afternoon profiles (Figures $10 \mathrm{a}$ and 10d) display a qualitatively excellent match between observations and model predictions.

[40] Concluding, the model calculations suggest that the entrainment into the TL is the key mechanism governing the TL dynamics. In particular, the entrainment flux balances the salinity stratification in the TL and is effective in removing heat from the TL.

\section{Discussion of the Turbulent Kinetic Energy Budget}

\subsection{Available Potential Energy}

[41] In many geophysical convective regimes, the bouyant production of TKE takes place at the interfaces of the convective layer (e.g., bottom heating/surface cooling of water above temperature of maximum density). Unlike such regimes, the radiatively driven convection is governed by buoyant TKE production in the interior of the CL. Hence not only the surface buoyancy flux, but the complete profile of the internal bouyancy flux $J(z)$ has to be considered for TKE scaling (where $J(z)$ is bouyancy parameter times the vertical turbulent temperature flux, see equation (8)). In our case, two conditions are met, allowing the internal production of buoyancy flux by radiative heating. First, the absorption of radiation decreases with depth, and second the bulk temperature $\bar{T}$ of the CL is below the temperature of maximum density. Thus the absorption of radiation leads to a destabilization of the water column. However, the second condition limits the occurrence of radiatively driven convection in time, as the absorption of radiation causes the CL to heat up.

[42] Compared to convection in a top mixed layer driven by surface cooling, the radiatively driven convection under ice is less efficient in using surface radiation flux for the generation of TKE. To illustrate this reduced efficiency, we trace the nonutilized portions of surface radiation flux $I_{0}$ in several steps (Figure 11, top panel).

[43] 1. As a reference regime for our comparison, we choose a convective top mixed layer driven by surface cooling. Such a regime features a buoyancy flux dropping to a good approximation linearly from the surface buoyancy flux $J_{0}$ at the surface to zero at the base of the CL [e.g., Mason, 1989, Figure 14]. Neglecting entrainment at the base of the CL, the mean generation rate of potential energy is given by half the surface buoyancy flux

$$
\bar{J}=\left(1-\frac{1}{2}\right) \beta_{0} I_{0}=\frac{1}{2} J_{0} .
$$

[44] 2. Given the stratification setup of radiatively driven convection under ice, the TL accumulates part of the surface radiation flux $I_{0}$. But the strong stratification in the TL does not allow the bouyant production of TKE, thus leaving behind only the TL/CL interfacial buoyancy flux $J\left(z_{1}\right)$ to govern the maximum possible generation rate of potential energy for a mixed CL,

$$
\bar{J}=\left(1-\frac{1}{2}\right) \bar{\beta} I\left(z_{1}\right)=\frac{1}{2} J\left(z_{1}\right) .
$$

In our case, around $50 \%$ of the surface radiation flux $I_{0}$ is spent to accumulate heat in the TL, and is subsequently not available for the production of TKE. This percentage features a significant daytime variation between $30 \%$ (in the afternoon) and $70 \%$ (in the morning), corresponding to the dynamics of $z_{1}$ (Figure 8).

[45] 3. Considering the actual vertical absorption pattern of radiation as parameterized by equations (3) and (4), the effective generation rate of potential energy is only a fraction of the above given maximum value (Figure 11, top panel). This fraction is commonly referred to as generation rate $\bar{J}^{\mathrm{APE}}$ of available potential energy (APE), as it is available for the production of TKE. $\bar{J}^{\mathrm{APE}}$ can be approximated on the basis of a simplified stratification: (1) starting with a mixed CL, (2) potential energy is generated by the absorption of radiation for an infinitesimal 


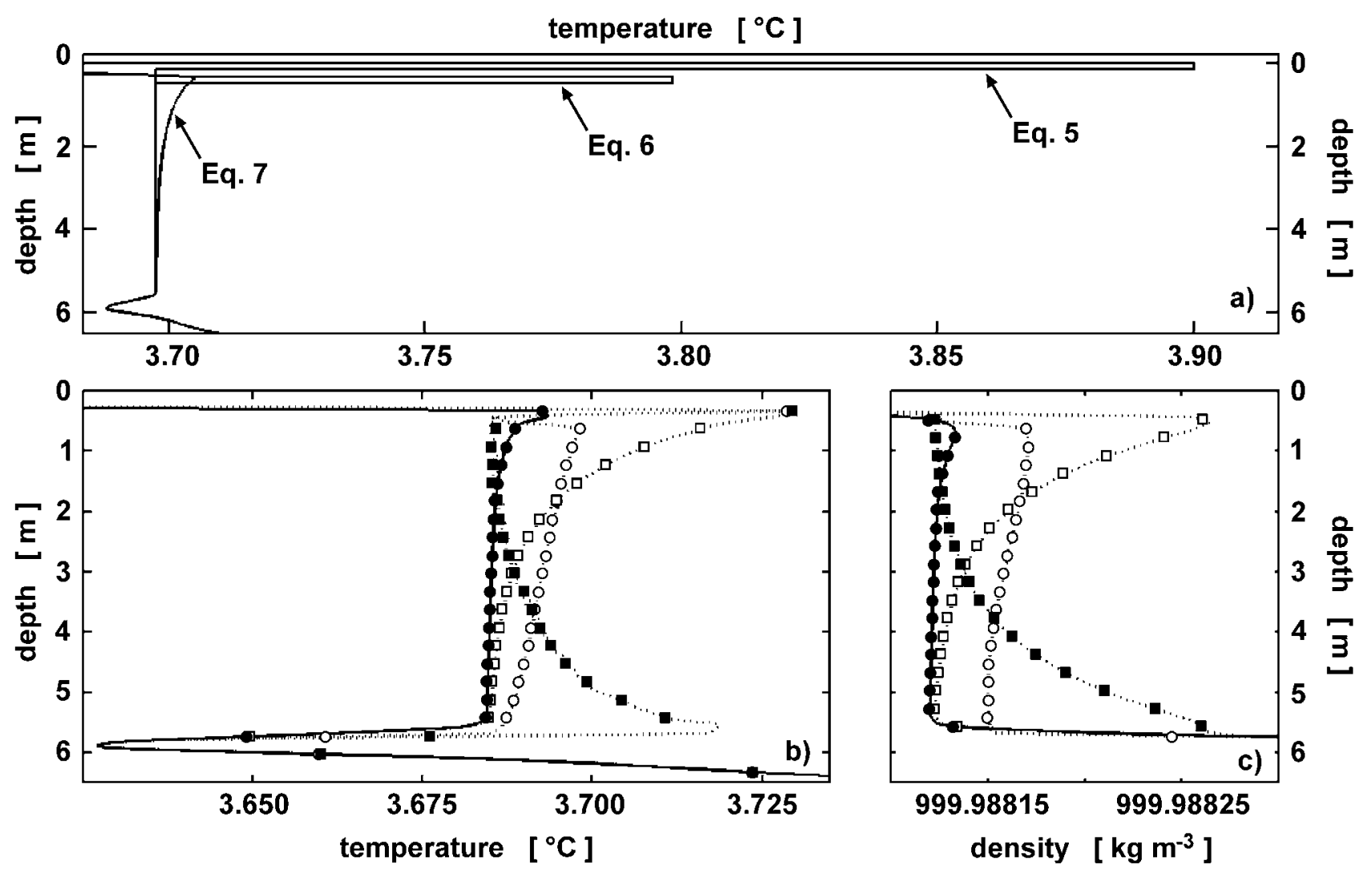

Figure 11. Illustration of the concept of available potential energy for different scenarios (section 5.1). The top panel displays the temperature stratification that would result from heat accumulation without convective adjustment processes: equation (5) by a surface heat flux, equation (6) by a top layer/ convective layer interfacial heat flux with radiative heat accumulation in the top layer, and equation (7) by the actual volumetric heating. The available potential energy is that part of the potential energy which is released when convective adjustment procedures eradicate density instabilities. The two bottom panels display a realistic initial stratification (solid circles on solid line), heat accumulation as in first panel case equation (7) (open squares on dotted line), plus two different convective reactions to density instabilities: according to model run $\Pi$ (solid squares on dotted line), and according to run $\Pi+\Pi^{*}+\Pi^{* *}$ (open circles on dotted line). A convective adjustment procedure would generate a mixed convective layer (not shown). The results as obtained by model run $\Pi+\Pi^{*}+\Pi^{* *}$ correspond best to observations.

time step, and (3) partially released by reinforcing a mixed CL. The difference in the potential energy content of the stratification in (2) and (3) per time leads to

$$
\begin{aligned}
\bar{J}^{\mathrm{APE}}= & \frac{\bar{\beta}}{z_{2}-z_{1}}\left[\int_{\mathrm{CL}}\left(z_{\mathrm{ref}}-z\right) \frac{\partial I}{\partial z} d z\right. \\
& \left.-\left(\frac{I\left(z_{2}\right)-I\left(z_{1}\right)}{z_{2}-z_{1}}\right) \int_{\mathrm{CL}}\left(z_{\mathrm{ref}}-z\right) d z\right] \\
= & \frac{\bar{\beta}}{z_{2}-z_{1}} \int_{\mathrm{CL}} I d z-\frac{\bar{\beta}}{2}\left(I\left(z_{1}\right)+I\left(z_{2}\right)\right),
\end{aligned}
$$

where $z_{\text {ref }}$ is an arbitrary reference depth for the evaluation of the potential energy. The expression in equation (7) is consistent with the generation rate of TKE as derived by Mironov et al. [2002, $B_{R}$ in equation (8)]. The maximum value in equation (6) represents the limiting case of $\bar{J}^{\mathrm{APE}}$ for infinite absorption in the CL (i.e. all radiation penetrating the TL is absorbed at $z_{1}$ ). The volumetric nature of the actual absorption pattern (as parameterized by equations (3) and (4)) leads to a further reduction in the efficiency of the use of surface radiation flux for the generation of TKE. In our case $\bar{J}^{\mathrm{APE}}$ is only about $58 \%$ of $0.5 J\left(z_{1}\right)$, so this effect is significant as well.

[46] For the estimates given by equations (5)-(7), a mixed $\mathrm{CL}$ is utilized as the reference stratification to define the available fraction of generated potential energy. But in reality, the regime may feature a slightly unstable stratification. For this case, model calculations demonstrate the absorption-driven generation of potential energy and its release for different system responses to unstable stratification (Figure 11, two bottom panels). In the unrealistic border case of an adiabatic nonmixing stabilization of the water column (model case $\Pi$ ), more potential energy is being released than with neutralizing the stratification to a mixed CL (adjustment procedure). However, according to CTD recordings, the quasiconservation of the slightly unstable stratification is most realistic (model case $\Pi+\Pi^{*}+\Pi^{* *}$ ). 
[47] The rate $\bar{J}^{\mathrm{APE}} / 0.5 J_{0}$ describes the regime's efficiency at utilizing surface radiation flux for the generation of TKE relative to our reference equation (5). It is chiefly governed by the total absorption of radiation in the TL and by the vertical gradient of the radiation flux $I$ in the upper CL. Unfortunately, it is not obvious how this efficiency is related to the vertical distribution of turbidity and to the ice-melting rate, as not only the extinction in the TL but also the TL thickness depends on these properties.

[48] The salinity stratification has an important impact on the total absorption of radiation in the TL by governing the TL thickness (sections 4.2 and 4.3). Thus the difference in bulk CL salinity and meltwater salinity, as well as the meltwater inflow rate $r_{m}$ are key properties codetermining the generation rate of TKE. Notice, though that salinity will not explicitly appear in any of the scaling relations (equations (10) and (11)), it is implicitly included via $I(z, t)$ and $z_{1}$.

\subsection{Generation of the Turbulent Kinetic Energy}

[49] The generation rate $J^{\mathrm{TKE}}$ of TKE allows an appropriate scaling of the TKE budget. The generation rate $\bar{J}^{\mathrm{APE}}$ provides a first approximation of $\bar{J}^{\mathrm{TKE}}$. Since this estimation is based on a simplified mixed layer parameterization of the $\mathrm{CL}$, its temporal dynamics is mainly bound to the surface radiation flux $I_{0}(t)$, the vertical position of the CL boundaries (particularly $z_{1}$ ), and the bulk buoyancy parameter $\bar{\beta}$. Thus thermistor string data of a high vertical resolution should allow rough estimates of $\bar{J}^{\mathrm{TKE}}$, provided $I\left(z_{1}\right)$ and $I\left(z_{2}\right)$ can be evaluated.

[50] Fortunately, in our case microstructure profiles are available on a regular basis. This allows alternatively calculating $J^{\mathrm{TKE}}$ on the basis of these consecutive profiles. The estimation is based on the transfer equation of temperature in the $\mathrm{CL}$, which reads

$$
\frac{\partial T}{\partial t}=-\frac{\partial Q}{\partial z}-\frac{\partial I}{\partial z},
$$

where $Q$ is the vertical turbulent temperature flux, $J=\beta Q$ is the buoyancy flux, and the molecular heat flux has been reasonably neglected. Multiplying equation (8) with $\beta$ and integrating from a reference depth $z_{\text {ref }}$ in the QL, where $Q\left(z_{\text {ref }}\right)=0$, to $z$ provides a measure of the buoyancy flux in terms of depth $z$

$$
J(z)=-\int_{z_{\mathrm{ref}}}^{z} \beta\left(z^{\prime}\right) \frac{\partial I}{\partial z^{\prime}}\left(z^{\prime}\right) d z^{\prime}-\int_{z_{\mathrm{ref}}}^{z} \beta\left(z^{\prime}\right) \frac{\partial T}{\partial t}\left(z^{\prime}\right) d z^{\prime},
$$

assuming that $\beta \partial Q / \partial z \approx \partial J / \partial z$. Averaging over the CL we get

$\bar{J}^{\mathrm{TKE}}=-\frac{1}{z_{2}-z_{1}} \int_{\mathrm{CL}}\left[\int_{z_{\mathrm{ref}}}^{z} \beta\left(z^{\prime}\right) \frac{\partial I}{\partial z^{\prime}}\left(z^{\prime}\right) d z^{\prime}+\int_{z_{\mathrm{ref}}}^{z} \beta\left(z^{\prime}\right) \frac{\partial T}{\partial t}\left(z^{\prime}\right) d z^{\prime}\right] d z$

[51] Contrary as with $\bar{J}^{\mathrm{APE}}$, the mixed layer assumption is applied only implicitly for the estimation of $\bar{J}^{\mathrm{TKE}}$ by assuming that $\beta \partial Q / \partial z \approx \partial J / \partial z$. This assumption is, however, hardly significant, given that $\partial Q / \partial z$ varies systematically and strongly with $z$, while $\beta$ does so only randomly and slightly (if $\bar{T}$ is not too close to the temperature of maximum density, as is the case on day 4). As $\bar{J}^{\mathrm{TKE}}$ is calculated from successive microstructure profiles, some scatter has to be accepted due to disturbances by laterally advected temperature inhomogeneities. But, in turn, the estimation is independent of an explicit assumption about the stratification in the CL and therefore is representative of the actual stratification. $\bar{J}^{\mathrm{TKE}}$ will be used to scale the TKE budget in the following. As scaled depth $\zeta$, we employ

$$
\zeta=\frac{z-z_{1}}{z_{2}-z_{1}}
$$

[52] For the shear-free CL, the TKE budget is given as

$$
\frac{d}{d t} \mathrm{TKE}=J-\varepsilon-\frac{\partial}{\partial z} F,
$$

where $F$ is the vertical transport of TKE. Two of the budget terms are directly available, i.e., $J$ and $\varepsilon$. As some averaging is required to allow statistically significant statements, we first look at the temporally averaged TKE budget in terms of $z$, and then consider the temporal dynamics of the CLaveraged TKE budget.

\subsection{Time-Averaged Turbulent Kinetic Energy Budget}

[53] Figure 12 displays the buoyancy flux $J(z)$ and the dissipation rate $\varepsilon$ of TKE both averaged over eight midday profiles and scaled with $\bar{J}^{\mathrm{TKE}}$ and $\zeta$. As we may assume a temporary steady state at midday (Figure 13), we approximate the divergence of the transport term $\partial F / \partial z$ as the residual of $J$ and $\varepsilon$ (Figure 12, heavy dotted line). Except for a transition area in the upper CL, all three terms of the TKE budget turn out qualitatively and quantitatively similar to LES results of shear-free convective boundary layers [Mason, 1989, Figure 14], and [Mironov et al., 2000 , Figure 11a], if with respect to our scale $\bar{J}^{\mathrm{TKE}}$ the LES results are rescaled with $0.5 J_{0}$ instead of $J_{0}$. The transition from high buoyancy fluxes and dissipation rates in the upper CL to zero $J$ and $\varepsilon$ in the TL is qualitatively reasonable as it reflects in the corresponding transition of the density gradient (Figure 11, bottom right panel, case $\left.\Pi+\Pi^{*}+\Pi^{* *}\right)$. Moreover, D. V. Mironov et al. (manuscript in preparation, 2003) present qualitatively similar results for corresponding LES of radiatively driven convection. However, a quantitative analysis remains impossible with no salinity data available to include in equation (9).

[54] The dissipation rate $\varepsilon$ of TKE features a rather homogenous distribution within the CL with a (maybe accidental) slight tendency to increase toward the EL, contrary to the opposite case for surface-flux driven convective boundary layers [e.g., Shay and Gregg, 1986]. The average $\varepsilon$ reaches $74 \%$ of the generation rate $\bar{J}^{\mathrm{TKE}}$. Dissipation of the same level indicates significant entrainment into the EL. Though our previous results suggest entrainment as well into the TL (sections 4.2 and 4.3), no significant dissipation was detected there. However, as the entrainment is likely to be restricted to a few centimeters at 


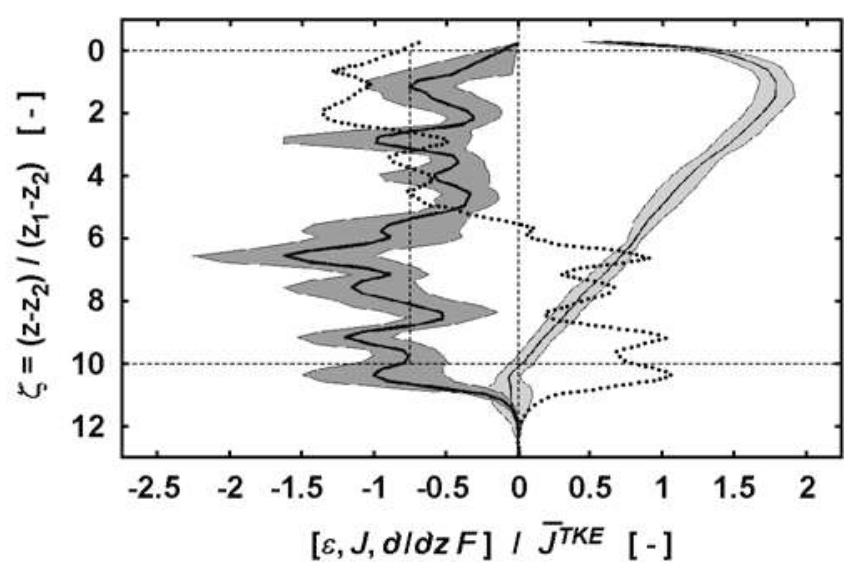

Figure 12. Vertical profiles of TKE budget terms averaged over eight midday profiles. Dissipation rate $\varepsilon$ of TKE with \pm 0.5 standard deviation from temporal averaging (thick solid line, dark shaded background); buoyancy flux $J$ with \pm 1 standard deviation from temporal averaging (thin solid line, light shaded background); divergence of the transport term $\partial F / \partial \mathrm{z}$ approximated as residual of $J$ and $\varepsilon$ (heavy dotted line). The mean scaled dissipation rate $\bar{\varepsilon}(t) / \bar{J}^{\mathrm{TKE}}(t-\Delta t)$ is $74 \%$. The ratio of total dissipation in the convective layer to total dissipation in the entrainment layer is 89:11. the base of the TL only, the microstructure method is not capable of resolving the phenomenon.

[55] The buoyancy flux $J$ displays a similar vertical pattern as found for surface-flux driven convective boundary layers [Lenshow et al., 1980; Mason, 1989; Mironov et al., 2000]. Besides qualitative differences in the upper $\mathrm{CL}$ as already discussed, the bouyant destruction of TKE below $\zeta=1$ is found less pronounced in comparison to results of the three LES studies mentioned above. Notice however, that the destruction of TKE by buoyancy in the EL is probably underestimated, as equation (9) is not accounting for the salinity that cogoverns the density stratification in this layer. Likewise, we expect erroneous estimates of $J(z)$ in the TL and likely no TKE is produced above $\zeta=0$.

[56] The transport $F$ removes TKE from areas with high production to areas with low production. It subsequently causes TKE to be homogeneously distributed within the CL.

[57] Referring to the total generation of TKE in the CL as $100 \%$, we estimate in the CL $74 \%$ dissipation, and in the EL $9 \%$ dissipation plus further $1.5 \%$ destruction by buoyancy. Hence the budget closes with a residual of $15 \%$ only, where the underestimation of the destruction of TKE by buoyancy in the EL may account for part of the residual. If not accidental, this excellent match confirms the accuracy of the absolute magnitude of the generation and dissipation

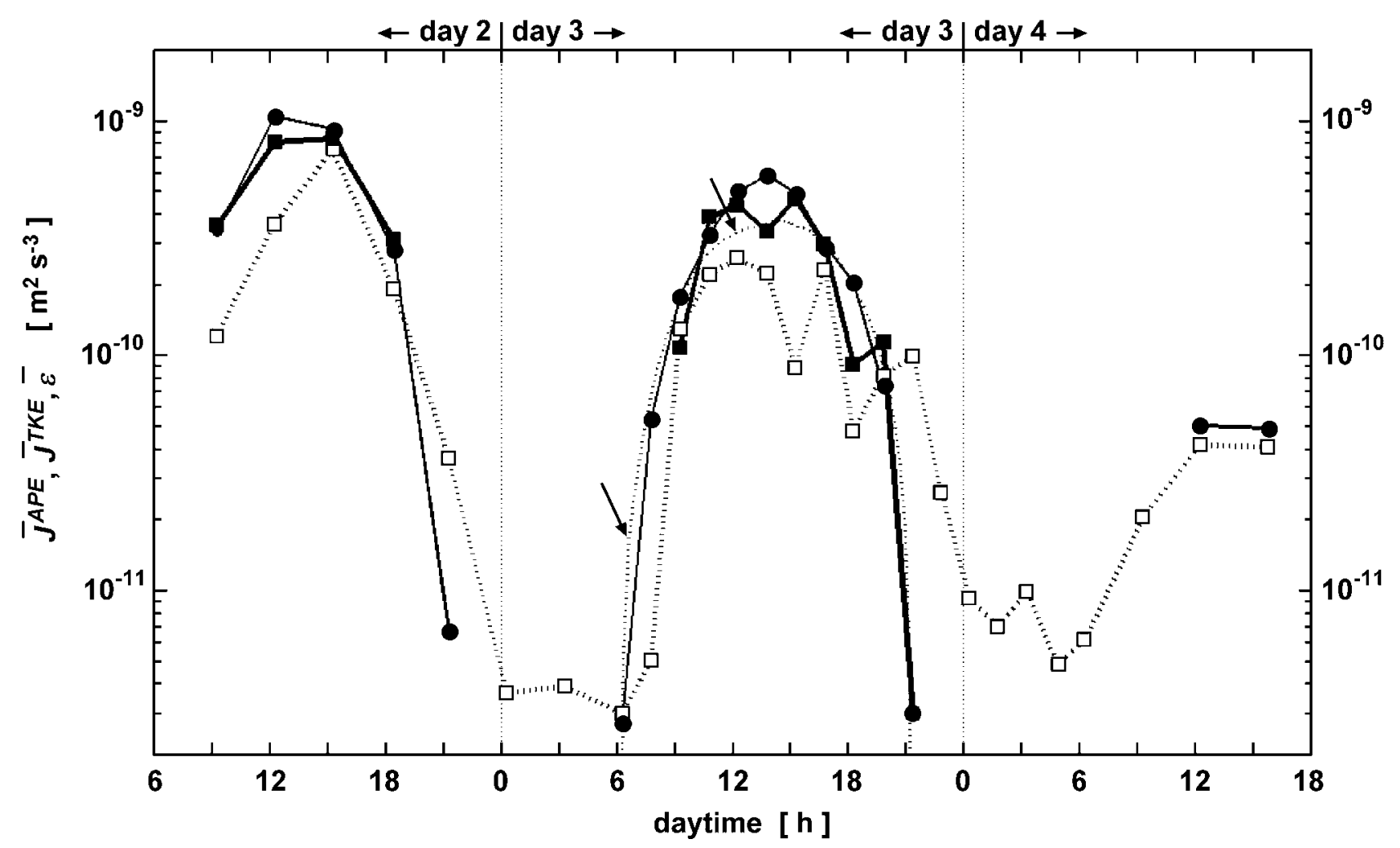

Figure 13. Generation rate $\bar{J}^{\mathrm{APE}}$ of available potential energy, generation rate $\bar{J}^{\mathrm{TKE}}$ of turbulent kinetic energy and dissipation rate $\bar{\varepsilon}$ of turbulent kinetic energy. All estimates are averaged over the convective layer. The generation rate $\bar{J}^{\mathrm{APE}}$ (solid circles on thin solid line) is calculated according to equation (7); $\bar{J}^{\mathrm{TKE}}$ (solid squares on thick solid line) is calculated according to equation $(10) ; \bar{\varepsilon}$ (open squares on thick dotted line) originates from temperature microstructure measurements. The arbitrarily scaled surface radiation flux $I_{0}$ is given as a reference (thin dotted line, see arrows). 


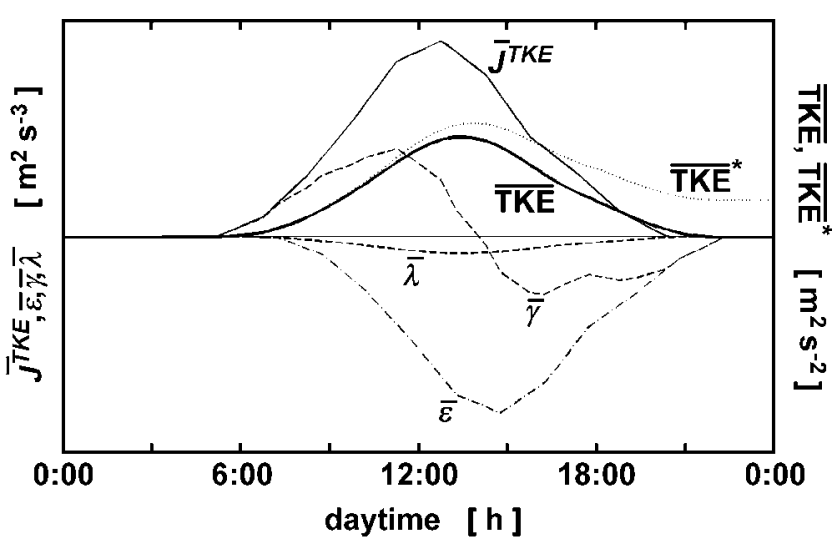

Figure 14. Schematic diurnal dynamics of the TKE budget equation (12) averaged over the convective layer. Generation rate $\bar{J}^{\mathrm{TKE}}$ and dissipation rate $\bar{\varepsilon}$ of TKE according to evaluation from microstructure data; a preliminary estimation of the temporal change rate $\bar{\gamma}$ of TKE is obtained as residual of $\bar{J}^{\mathrm{TKE}}$ and $\bar{\varepsilon}$; temporally integrating $\bar{\gamma}$ results in a preliminary estimate of the turbulent kinetic energy $\overline{\mathrm{TKE}}^{*}$. Assumptions (section 5.4) on the export rate $\vec{\lambda}$ of TKE allow achieving a better estimate of $\overline{\mathrm{TKE}}$, which consistently is zero during nights.

rate estimates. As the estimation of $J(z)$ is sensitive to the parameterization of $I(z)$, we may further conclude that equation (4) yields an appropriate parameterization of the absorption characteristics.

\subsection{Depth-Averaged Turbulent Kinetic Energy Budget}

[58] The diurnal dynamics of $\bar{J}^{\mathrm{APE}}, \bar{J}^{\mathrm{TKE}}$, and $\bar{\varepsilon}$ are shown in Figure 13. With only one profile during the day, day 1 was excluded from the Figure. It is noteworthy to mention that none of the data shown in Figure 13 are time averaged in any respect. Only $\bar{J}^{\mathrm{TKE}}$ is remapped onto the common time axes. The generation and dissipation rate of TKE display a temporal pattern similar to the surface radiation flux (Figure 13, curve of $I_{0}$ for day 2). But while $\bar{J}^{\mathrm{TKE}}$ follows the dynamics of $I_{0}$ instantaneously, $\bar{\varepsilon}$ lags, on average, by approximately 1.5 hours. The values of $\bar{J}^{\mathrm{APE}}$ and $\bar{J}^{\mathrm{TKE}}$ are practically congruent, suggesting that the mixed layer assumption is not a major limitation when considering scaling of radiatively driven convection in ice-covered lakes. However, $\bar{J}^{\mathrm{TKE}}$ and $\bar{\varepsilon}$, the estimations of which are based upon the consecutive microstructure profiles, display some temporal scatter, contrary to $\bar{J}^{\mathrm{APE}}$, which is derived from the mixed layer parameterization. All properties shown in Figure 13 display a superordinate declining trend with time, because of $\beta$ decreasing as $\bar{T}$ approaches the temperature of maximum density. Looking at the nighttime dissipation rates, we conclude that the methodical goal of resolving rates down below $10^{-11} \mathrm{~m}^{2} \mathrm{~s}^{-3}$ has been achieved. The increasing amplitude of temperature finestructure scatter when approaching the temperature of maximum density has obviously resulted in a slightly increased noise floor of the estimations (see night of day $3 /$ day 4 ).

[59] The diurnal dynamics of $\bar{J}^{\mathrm{TKE}}$ and $\bar{\varepsilon}$ allow deducing rough estimates of the dynamics of the temporal change rate $\bar{\gamma}$ of TKE and export rate $\bar{\lambda}$ of TKE (export from CL), where

$$
\bar{\gamma}=\frac{1}{z_{1}-z_{2}} \int_{\mathrm{CL}} \frac{\partial \mathrm{TKE}}{\partial t} d z
$$

and

$$
\bar{\lambda}=\frac{1}{z_{2}-z_{1}} \int_{\mathrm{CL}} \frac{\partial F}{\partial z} d z=\frac{F\left(z_{2}\right)-F\left(z_{1}\right)}{z_{2}-z_{1}} .
$$

The depth-resolving TKE budget (section 5.3) reveals that, on temporal average, only around $11 \%$ of the generated TKE is transported from the CL to the EL. Moreover, we assume no significant transport into the TL. Given that, we may in a first step approximate $\bar{\gamma}$ as the residual of $J$ and $\varepsilon$ (Figure 14, dashed line), neglecting the export rate $\bar{\lambda}$ for the moment. By temporally integrating $\bar{\gamma}$, we receive a first rough estimate of the diurnal dynamics of TKE $^{*}$ (Figure 14, dotted line). To close the budget, the temporal dynamics $\bar{\lambda}$ has to be considered. As the stability of the EL remains practically constant during the day (Figure 7 and section 4.1), we may assume that $\bar{\lambda}$ roughly follows the dynamics of TKE i.e., the transport $F\left(z_{2}\right)$ is proportional to the abundance of $\overline{T K E}$ in the CL. With the necessity to meet TKE = 0 after the course of the day, $\bar{\lambda}$ can be determined, allowing the recalculation of $\bar{\gamma}$ and subsequently TKE (Figure 14, thick solid line). The TKE budget reveals that in a CLaveraged perspective, the change rate of TKE is as significant as its generation and dissipation rate. Therefore we suggest introducing the time lag $\Delta t$ between generation and dissipation for scaling individual profiles of $\varepsilon$, i.e., to scale $\varepsilon(t)$ with $\bar{J}^{\mathrm{TKE}}(t-\Delta t)$ especially if profiles from the earlier forenoon or the later afternoon are considered.

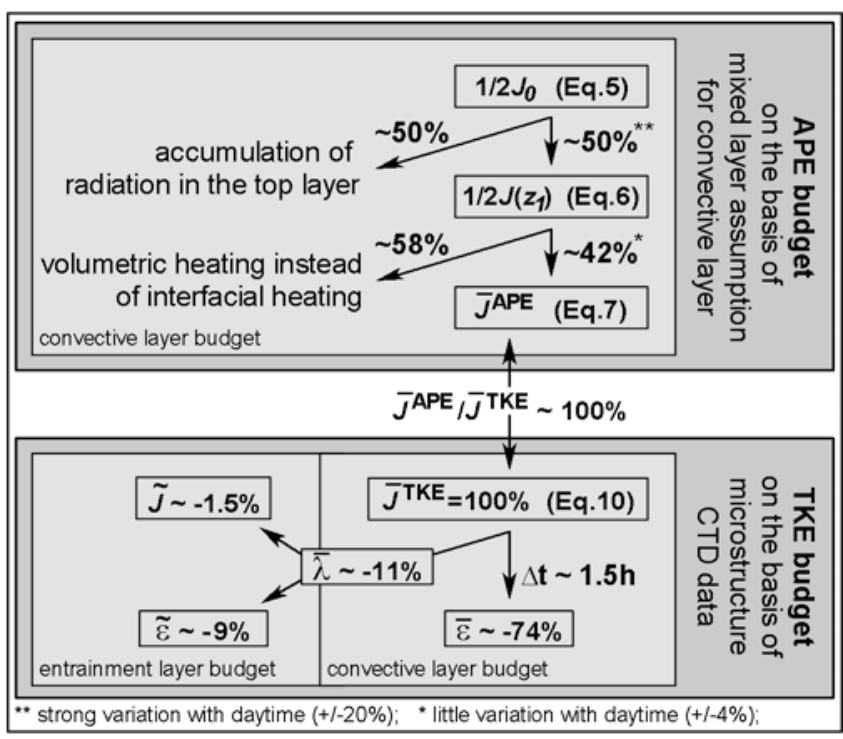

Figure 15. Layer averaged budget of available potential energy APE and turbulent kinetic energy TKE. See section 5 for further explanation. 
[60] Summarizing, a layer-averaged TKE budget is assembled in Figure 15, including measures of the efficiency of utilizing surface radiation flux for the generation of TKE. The budget is representative of the daytime situation.

\section{Summary and Conclusions}

[61] Radiatively driven convection in an ice-covered lake has been studied employing a temperature microstructure profiler, a submersible radiation logger, a CTD profiler, and a string of high-precision thermistors. The stratification of the water column beneath the ice is characterized by a system of five distinct layers. During the day, two thin layers enclose the quasi-mixed convective layer (CL). These enclosing layers both feature a dominant stabilizing salinity gradient and a destabilizing temperature gradient and are subject to considerable entrainment from the CL. The dynamics of the two interfaces between the $\mathrm{CL}$ and the enclosing layers are chiefly governed by the entrainment and display a diurnal cycle. On a multiday timescale, the CL extends only downward. This three-layer system is further embedded by a thin but extremely stable layer on top (directly below the ice) and by a quiescent layer (QL) below.

[62] Radiatively driven convection generates buoyancy flux directly inside the CL, contrary, e.g., to coolinginduced convection in a surface boundary layer. This circumstance results in a considerably reduced production of available potential energy (APE) (by $58 \%$ in our case), relative to convection driven by a surface flux. Moreover, a significant portion of the surface radiation flux $(50 \%$ in our case) is absorbed in the stable top layer (TL) and is thus not available for the generation of turbulent kinetic energy (TKE) either. Hence the generation rate of TKE is considerably dependent on the absorption characteristics and the TL thickness, which in turn reflect the salinity stratification and the melting rate of the ice cover.

[63] Consecutive temperature microstructure profiles allow direct estimates of the generation and dissipation rates of TKE. Two budgets of the TKE are presented. The temporally averaged budget reveals a vertical distribution of generation and dissipation rates similar to the case of cooling-induced convection in a surface boundary layer. However, as prominent difference, a transition layer was found in the upper CL, where both rates drop back to zero toward the layer on top. Approximately, $75 \%$ of the TKE that is generated in the CL was found to dissipate in the same layer, while another $10 \%$ is transferred to the entrainment layer (EL) below and dissipated there. The small overall residual of the independently derived generation and dissipation rates indicates the excellent quality of their estimations in spite of the low turbulence level (generation/ dissipation rates below $10^{-9} \mathrm{~W} \mathrm{~kg}^{-1}$ ).

[64] The second TKE budget is spatially averaged over the $\mathrm{CL}$ but resolves the diurnal dynamics of the budget terms. The generation rate and the dissipation rate of TKE display a close correlation. Their temporal dynamics are quasicongruent but the dissipation lags, on average, 1.5 hours behind the generation. The remaining terms, the temporal change rate and the export rate of TKE, are estimated with the help of an assumption on the latter term.
The change rate is found in the same order of magnitude as generation rate and dissipation rate, while the export rate seems less significant. A scaling for the TKE budget is suggested, which accounts for these special circumstances.

\section{Appendix A: Numerical Realization of the Model Processes}

[65] In the following, the superscripts denote the temporal index $\tau$, and the subscripts denote the grid node index $\delta$. The time step is denoted by $\Delta t$, which passes while $\tau$ increases by $1(\Delta t=150 \mathrm{~s}$, section 2.3). Likewise, $\Delta z$ is the grid size, the distance between node $\delta$ and $\delta+1(\Delta z=0.5 \mathrm{~mm}$, section 2.3). At the surface $\delta=1$ and increases downward. To address the different procedures during one time step, the temporal index $\tau$ is subdivided (e.g., $\tau, \tau+0.2, \tau+0.4$ ). Notice, however, that $\Delta t$ remains constant and in particular is not subdivided together with $\tau$.

[66] As the first action per time step, the accumulation of radiation is taken into account

$$
T_{\delta}^{\tau+0.2}=T_{\delta}^{\tau}-\frac{\partial I}{\partial z}(t, z) \Delta t
$$

where the divergence of the radiation flux $I$ is calculated from equations (3) and (4). To maintain the boundary conditions at the ice/water interface (section 3.1)

$$
\begin{gathered}
T_{\delta=1}=0^{\circ} \mathrm{C}, \\
S_{\delta=1}=0.002 \mathrm{psu} .
\end{gathered}
$$

Therefore equation (A1) is only applied to cells with grid node index $\delta>1$

[67] As the second procedure per time step, meltwater is released into the water column. Due to its density being smaller than anywhere else in the water column, the meltwater stratifies on top. Since the vertical grid is defined relative to the underside of the ice, the water column is shifted downward according to the meltwater inflow rate $r_{m}$ by

$$
d z=r_{m}(t) \Delta t
$$

Notice, that $d z \ll \Delta z$. To maintain the grids positioning, the contributions of $d z$ are preliminarily only summed for as many time steps, until the water column can be shifted downward a complete cell

$$
\begin{aligned}
& T_{\delta+1}^{\tau+0.4}=T_{\delta}^{\tau+0.2}, \\
& S_{\delta+1}^{\tau+0.4}=S_{\delta}^{\tau+0.0} .
\end{aligned}
$$

And to maintain the grids size, the undetermined additional grid node on top is set according to equation (A2), while the last grid node on the bottom is cropped.

[68] Molecular diffusion is split up to work at two separate stages of the numerical progress, each of which is proportional to $0.5 \Delta t$. Moreover, a 1000 -fold time split- 
ting is employed to ensure the numerical stability of the diffusive process

$$
\begin{aligned}
& T_{\delta}^{\tau+i}=T_{\delta}^{\tau+i-1}+\kappa_{T} \frac{T_{\delta+1}^{\tau+i-1}-2 T_{\delta}^{\tau+i-1}+T_{\delta-1}^{\tau+i-1}}{(\Delta z)^{2}} \frac{\Delta t}{1000}, \\
& S_{\delta}^{\tau+i}=S_{\delta}^{\tau+i-1}+\kappa_{S} \frac{S_{\delta+1}^{\tau+i-1}-2 S_{\delta}^{\tau+i-1}+S_{\delta-1}^{\tau+i-1}}{(\Delta z)^{2}} \frac{\Delta t}{1000},
\end{aligned}
$$

with $i=0.4+0.2 j / 500$ and $j=\{1 \ldots .500\}$ as the third procedure per time step.

[69] The numerical procedure to react on density instabilities constitutes the core of the model. Contrary to common adjustment procedures, we employ an adiabatic, nonmixing stabilizing procedure, and allow only the subsequent diffusion process to perform mixing. This provides a gain as outlined in section 5.1. In the following, one basic stabilizing procedure and two additional procedures for further enhancements are considered.

[70] The basic stabilizing procedure $\Pi$ is defined as a permutation of the set of grid node indices $\delta$. This permutation is represented by a displacement vector $\mathbf{d}_{\delta}^{\mathrm{\tau}}$ which states for each cell (referred to by a unique grid node index $\delta$ ) a displacement, due from $\tau+0.6$ to $\tau+0.8$. The displacements are expressed as change in $\delta$, such that e.g., $\mathbf{d}_{\delta=3}=5$ indicates, the cell at node $\delta=3$ is to be displaced to node $\delta=8$. The basic stabilizing procedure then reads

$$
\begin{aligned}
& T_{\delta}^{\tau+0.8}=T_{\delta+\mathbf{d}_{\delta}^{\tau}}^{\tau+0.6}, \\
& S_{\delta}^{\tau+0.8}=S_{\delta+\mathbf{d}_{\delta}^{\tau}}^{\tau+0.6} .
\end{aligned}
$$

The displacement vector $\mathbf{d}$ shall ensure that the entire water column gains a completely stable stratification. We therefore implicitly define $\mathbf{d}$ by the commensurate condition

$$
\rho\left(S_{\delta_{2}}^{\tau+0.8}, T_{\delta_{2}}^{\tau+0.8}\right)>\rho\left(S_{\delta_{1}}^{\tau+0.8}, T_{\delta_{1}}^{\tau+0.8}\right) \text { for any } \delta_{2}>\delta_{1} \text {. }
$$

[71] Two refinements of $\Pi$ are further introduced to generate a more realistic model of the convective response to density instabilities (Figures 10 and 11). The first refinement $\Pi^{*}$ delimits the water parcel displacement speed of the basic procedure $\Pi$. Therefore the entries of $\mathbf{d}_{\delta}$ are restrained by

$$
\begin{gathered}
\left|\mathbf{d}_{\delta}^{*}\right| \leq w_{R} d t \approx\left(1.25 \times 10^{-3} \mathrm{~ms}^{-1}+I_{0}(t) 16.8 \mathrm{~K}^{-1}\right) d t \\
w_{R}=\left[\left(z_{2}-z_{1}\right) \bar{J}^{\mathrm{APE}}\right]^{1 / 3},
\end{gathered}
$$

defining $\mathbf{d}_{z}^{*}$ as the restrained $\mathbf{d}_{z} ; w_{R}$ refers to the velocity scale as suggested by Mironov et al. [2002, equation (8)]. We employ a linear parameterization of the empirical relationship $w_{R}\left(I_{0}\right)$, raised for small surface radiation fluxes, to enable some restratification during the nights (right-hand side of equation (A8a)). The manipulation equation (A8a) causes the displacement vector $\mathbf{d}_{\delta}^{*}$ to violate the requirement of $\Pi^{*}$ being a permutation of the set of grid node indices $\delta$. We therefore manually reinforce this condition, under minimizing of the maximum necessary adaptation to an entry of $\mathbf{d}_{\delta}^{*}$. In case of also incorporating the second refinement into the model, this step only follows at the end of the second refinement.

[72] The second refinement $\Pi^{* *}$ enables penetrative entrainment by artificially enlarging the displacements. The excess displacement (overshooting) is realized by adding a small portion $f$ of the cell's displacement from the preceding time step to its actual displacement

$$
\mathbf{d}_{\delta}^{\tau * *}=\mathbf{d}_{\delta}^{\tau *}+f \mathbf{d}_{\delta+d_{\delta}^{\tau-1} * *}^{\tau-1} *
$$

As we presume that the penetration strength depends on the background density stratification, the factor $f$ was constructed in terms of the stability $N^{2}$ at the displacement target position of $\Pi^{*}$, where the Brunt-Väisälä frequency $N$ is evaluated from smoothed $T$ and $S$ profiles (running mean filtered with $0.05 \mathrm{~m}$ window). The observed temperature stratification suggests maximum penetration lengths of 0.01 $\mathrm{m}$ into the TL $\left(N^{2}\right.$ typically $=10^{-4}$ to $\left.10^{-3} \mathrm{~s}^{-2}\right)$, and of 0.1 $\mathrm{m}$ into the $\mathrm{EL}\left(N^{2}\right.$ typically $=10^{-6}$ to $\left.10^{-5} \mathrm{~s}^{-2}\right)$. Given the maximum penetration speed of approximately $0.002 \mathrm{~m} \mathrm{~s}^{-1}$ (equation (A8) with $\rho c_{p} I_{0}{ }^{\max } \approx 200 \mathrm{~W} \mathrm{~m}^{-2}$ and $\Delta t=150 \mathrm{~s}$ ), $f$ is constructed in logarithmic interpolation to meet the observations

$$
\begin{gathered}
N^{2} \leq 10^{-6} \mathrm{~s}^{-2}: f=\frac{0.1 \mathrm{~m}}{0.002 \mathrm{~ms}^{-1} \times 150 \mathrm{~s}}=\frac{1}{3}, \\
10^{-6} \mathrm{~s}^{-2}<N^{2} \leq 10^{-3} \mathrm{~s}^{-2}: f=-0.0434 \ln \left(N^{2} 464 \mathrm{~s}^{2}\right),
\end{gathered}
$$

$$
10^{-3} \mathrm{~s}^{-2}<N^{2}: f=\frac{0.01 \mathrm{~m}}{0.002 \mathrm{~ms}^{-1} \times 150 \mathrm{~s}}=\frac{1}{30},
$$

where equation (A10a) keeps $\Pi^{* *}$ from revoking the displacement speed's limitation by $\Pi^{*}$ in the neutrally stratified CL. The arbitrary form of parameterization equation (A10b) is, fortunately, barely of influence, because the entrainment into the DL and into the EL is governed by equations (A10c) and (A10a).

[73] Finally, as last action per time step, the second part of the molecular diffusion process is employed according to equation (A5) but with $i=0.8+0.2 j / 500$ and $j=$ $\{1 . .500\}$.

\section{Appendix B: Additional Details on the Ice Melting Process}

[74] Another indirect measure of the melting rate of the ice cover originates from the comparison of the microstructure profiles with the thermistor string data. While the depth readings of the profiler are calibrated relative to the ice/water interface, the thermistor string was fixed relative to the air/ice interface. Therefore matching the temperature of the thermistor $T\left(z_{0}\right)$, which was deployed in the molecular layer, with the consecutive temperature profiles $T(z)$ 
through the same layer allowed estimating the melting rate from $z-z_{0}$ with a temporal resolution given by the profiling rate (Figure 5, solid circles upon dotted line).

[75] This comparison-derived estimation is more precisely a measure of the rate of decrease of the cover thickness, while the budget-derived estimation is indeed a measure of the melting rate. The remarkable temporal relationship between these two measures resembles a signal with a delayed, temporally averaged response and likely accounts for the internal melting process. Decomposing ice may in fact lead to a delayed overall decrease of the ice thickness, as without ice-restructuring processes, internal melting would only lead to an increasing porosity of the ice. Hence the time lag between internal melting and the decrease of the cover thickness, in our case around 6 hours, represents a recrystallization timescale.

[76] From days 2 to 3, the comparison-derived decrease rate of the ice-cover thickness of $3.2 \mathrm{~cm} \mathrm{~d}^{-1}$ is practically identical to the corresponding value from the budgetderived melting rate. This finding confirms that the bulk of the melted ice cover effectively intrudes into the water column.

[77] Acknowledgments. We would like to thank P. Boyarinov, A. Mitrokhov, N. Palshin, M. Petrov, and M. Schurter for their effort during the field campaign, L. Bengtsson and N. Filatov for their organizational support, C. Schär and J. Sander for critically reviewing the manuscript, and D. McGinnis for his linguistic advice. This study was supported by the Swiss National Science Foundation, grants 20-50761.97 and 2000063723.00, as well as by the EU Commissions, grant INTAS-97-0734.

\section{References}

Barnes, D. F., and J. E. Hobbie, Rate of melting at the bottom of floating ice, U.S. Geol Surv. Prof. Pap., 400B, B392-B394, 1960.

Batchelor, G. K., Small-scale variations of convected quantities like temperature in turbulent fluid: I. General discussion and the case of small conductivity, J. Fluid Mech., 5, 113-133, 1959.

Bengtsson, L., Mixing in ice-covered lakes, Hydrobiologia, 322, 91-97, 1996.

Birge, E. A., The apparent sinking of ice in lakes, Science, 32, 81-82, 1910.

Brunt, D., Patterns in ice and cloud, Weather, 1, 184-185, 1946.

Dillon, T. M., and D. R. Caldwell, The Batchelor spectrum and dissipation in the upper ocean, J. Geophys. Res., 85, 1910-1916, 1980.

Farmer, D. M., Penetrative convection in the absence of mean shear, $Q . J$. R. Meteorol. Soc., 101, 869-891, 1975.

Gibson, C. H., and W. H. Schwartz, The universal equilibrium spectra of turbulent velocity and scalar fields, J. Fluid Mech., 16, 365-384, 1963.

Gloor, M., Methode der Temperaturmikrostruktur und deren Anwendung auf die Bodengrenzschicht in geschichteten Wasserkörpern, Ph.D. thesis, Eidg. Tech. Hochschule, Zürich, Switzerland, 1995.

Grant, H. L., B. A. Hughes, M. W. Vogel, and A. Moilliet, The spectrum of temperature fluctuations in turbulent flow, J. Fluid. Mech., 34, 423-442, 1968.

Gregg, M. C., The study of mixing in the ocean, a brief history, Oceanography, IV, 39-45, 1991.

Gregg, M. C., C. S. Cox, and P. W. Hacker, Vertical microstructure measurements in the central North Pacific, J. Phys. Oceanogr., 3, 458-469, 1973.
Ivey, G. N., and J. Imberger, On the nature of turbulence in a stratified fluid: I. The energetics of mixing, J. Phys. Oceanogr, 21, 650-658, 1991.

Kocsis, O., H. Prandke, A. Stips, A. Simon, and A. Wüest, Comparison of dissipation of turbulent kinetic energy determined from shear and temperature microstructure, J. Mar. Syst., 21, 67-84, 1999.

Lenshow, D. H., J. C. Wyngaard, and W. T. Pennell, Mean-field and second-moment budgets in a baroclinic, convective boundary layer, $J$. Atmos. Sci., 37, 1313-1326, 1980.

Litinskaya, K. D., and Y. Polyakov, Lakes of Vendyury group - Uros, Rindozero, and Vendyurskoe, in Water Resources of Karelia and Their Use, pp. 57-66, Karelian Branch of Acad. of Sci. of USSR, Petrozavodsk, Russia, 1975.

Malm, J., A. Terzhevik, L. Bengtsson, P. Boyarinov, A. Glinsky, N. Palshin, and M. Petrov, A field study of thermo- and hydrodynamics in three small Karelian lakes during winter 1994/1995, Rep. 3197, LUTVDG/ (TVVR-3197) 1-220/(1996), 220 pp., Dep. of Water Resour. Eng., Inst. of Technol., Univ. of Lund, Lund, Sweden, 1996.

Malm, J., A. Terzhevik, L. Bengtsson, P. Boyarinov, A. Glinsky, N. Palshin, and M. Petrov, Temperature and salt content regimes in three shallow icecovered lakes: I. Temperature, salt content, and density structure, Nord. Hydrol., 28, 99-128, 1997a.

Malm, J., A. Terzhevik, L. Bengtsson, P. Boyarinov, A. Glinsky, N. Palshin, and M. Petrov, Temperature and salt content regimes in three shallow icecovered lakes: II. Heat and mass fluxes, Nord. Hydrol., 28, 129-152, $1997 \mathrm{~b}$.

Mason, P. J., Large-eddy simulation of the convective atmospheric boundary layer, J. Atmos. Sci., 46, 1492-1516, 1989.

Mironov, D. V., V. M. Gryanik, C.-H. Moeng, D. J. Olbers, and T. H. Warncke, Vertical turbulence structure and second-moment budgets in convection with rotation: A large-eddy simulation study, Q. J. R. Meteorol. Soc., 126, 477-515, 2000.

Mironov, D. V., A. Yu. Terzhevik, G. B. Kirillin, T. Jonas, J. G. B. Malm, and D. M. Farmer, Radiatively driven convection in ice-covered lakes: Observations, scaling, and a mixed layer model, J. Geophys. Res., 107, 3032, doi:10.1029/2001JC000892, 2002.

Neumann, H. G., Zellmuster auf der Oberfläche eines Teiches, Contrib. Atmos. Phys., 30, 246-253, 1958.

Oakey, N. S., Determination of the rate of dissipation of turbulent energy from simultaneous temperature and velocity shear microstructure measurements, J. Phys. Oceanogr., 12, 256-271, 1982.

Petrov, M. P., and G. G. Sutyrin, Diurnal cycle of convection in an icecovered lake, Meteorol. Gidrol., 1, 91-98, 1984.

Price, A. G., and T. Dunne, Energy balance computations of snowmelt in a subarctic area, Water Resour. Res., 12, 686-694, 1976.

Sander, J., A. Simon, T. Jonas, and A. Wüest, Surface turbulence in natural waters: A comparison of large eddy simulation with microstructure measurements, J. Geophys. Res., 105, 1195-1207, 2000.

Shay, T. J., and M. C. Gregg, Convectively driven turbulent mixing in the upper ocean, J. Phys. Oceanogr., 16, 1777-1798, 1986.

Simon, A., Turbulent mixing in the surface boundary layer of lakes, Ph.D. thesis, ETH, Zürich, Switzerland, 1997.

Woodcock, A. H., Melt patterns in ice over shallow waters, Limnol. Oceanogr., 10, R290-R297, 1965.

T. Jonas, Alpine Environment Section, Swiss Federal Institute for Snow and Avalanche Research, Flüelastrasse 11, CH-7260 Davos Dorf, Switzerland. (jonas@slf.ch)

D. V. Mironov, Department of Meteorological Analysis and Modelling, German Weather Service, Postfach 100465, Frankfurter Strasse 135, D-63067 Offenbach am Main, Germany. (dmitrii.mironov@dwd.de)

A. Y. Terzhevik, Northern Water Problems Research Institute, Russian Academy of Sciences, Aleksander Nevsky Street 50, 185003 Petrozavodsk, Russia. (ark@nwpi.krc.karelia.ru)

A. Wüest, (corresponding author) Applied Aquatic Ecology, Limnological Research Center Kastanienbaum, EAWAG, Seestrasse 79, CH-6047 Kastanienbaum, Switzerland. (wuest@eawag.ch) 